\title{
Underwater Radiance Distributions Measured with Miniaturized Multispectral Radiance Cameras
}

\author{
David ANTOINe, ANDrÉ Morel, AND Edouard LEYMARIE \\ Laboratoire d'Océanographie de Villefranche, Centre National de la Recherche Scientifique, Villefranche-sur-Mer, \\ and Université Pierre et Marie Curie, Paris O6, Paris, France
}

\section{AMEl HOUYOU}

CIMEL Electronique, Paris, and Laboratoire d'Océanographie de Villefranche, Centre National de la Recherche Scientifique, Villefranche-sur-Mer, and Université Pierre et Marie Curie, Paris O6, Paris, France

\section{BERNARD GENTILI}

Laboratoire d'Océanographie de Villefranche, Centre National de la Recherche Scientifique, Villefranche-sur-Mer, and Université Pierre et Marie Curie, Paris, Paris O6, France

\section{Stéphane Victori, Jean-Pierre Buis, Nicolas Buis, Sylvain Meunier, MARIUS CANINI, AND DIDIER CROZEL}

CIMEL Electronique, Paris, France

Bertrand Fougnie AND PATRICE HENRY

Centre National d'Etudes Spatiales, Toulouse, France

(Manuscript received 2 December 2011, in final form 11 May 2012)

\begin{abstract}
Miniaturized radiance cameras measuring underwater multispectral radiances in all directions at highradiometric accuracy (CE600) are presented. The camera design is described, as well as the main steps of its optical and radiometric characterization and calibration. The results show the excellent optical quality of the specifically designed fish-eye objective. They also show the low noise and excellent linearity of the complementary metal oxide semiconductor (CMOS) detector array that is used. Initial results obtained in various oceanic environments demonstrate the potential of this instrument to provide new measurements of the underwater radiance distribution from the sea surface to dimly lit layers at depth. Excellent agreement is obtained between nadir radiances measured with the camera and commercial radiometers. Comparison of the upwelling radiance distributions measured with the CE600 and those obtained with another radiance camera also shows a very close agreement. The CE600 measurements allow all apparent optical properties (AOPs) to be determined from integration of the radiance distributions and inherent optical properties (IOPs) to be determined from inversion of the AOPs. This possibility represents a significant advance for marine optics by tying all optical properties to the radiometric standard and avoiding the deployment of complex instrument packages to collect AOPs and IOPs simultaneously (except when it comes to partitioning IOPs into their component parts).
\end{abstract}

Corresponding author address: David Antoine, Laboratoire d'Océanographie de Villefranche (LOV), Quai de la Darse, BP8, 06230 Villefranche-sur-Mer, France.

E-mail: antoine@obs-vlfr.fr

\section{Introduction}

The geometrical structure of the radiance distribution inside the upper layer of the ocean is primarily determined by the radiance distribution above the surface 
(resulting from the sun's position and the sky contribution) and then modified by reflection and refraction effects at the air-water interface. Progressing downward inside the water body, the radiant field is continuously modified by two interplaying phenomena: absorption by which photons disappear, and scattering by which photons are simply diverted from their initial direction of propagation. These phenomena are described by coefficients that belong to the "inherent" optical properties (IOPs; Preisendorfer 1961). The way these processes alter the radiant field is described by the radiative transfer equation (RTE) (Mobley 1994).

To describe the radiant field inside a scattering/ absorbing medium, like a water body, the spectral radiance $(L)$ is the fundamental radiometric quantity (units: $\mathrm{W} \mathrm{m} \mathrm{m}^{-2} \mathrm{sr}^{-1} \mathrm{~nm}^{-1}$ ); it is defined as the radiant power $(\Phi)$ in a specified direction $(\theta, \phi)$ per unit of solid angle $(\Omega)$, per unit area $(A)$, and within a wavelength interval $d \lambda$, centered on the wavelength $\lambda$ :

$$
L(\theta, \phi, \lambda)=d^{3} \Phi / d A d \Omega d \lambda,
$$

where $\theta$ and $\phi$ are a polar (zenith) angle and an azimuth angle defined in an appropriate coordinate system, the infinitely small area $d A$ is normal to the beam direction (i.e., normal to the direction that photons travel), and the infinitely narrow solid angle $d \Omega$ is centered on the direction that the photons travel. The ensemble of the radiance values $L(\Xi)$ in every direction of the whole space (in $\Xi=4 \pi \mathrm{sr}$ ) provides a complete description of the geometric structure of the radiant field. When only upwelling radiances are considered, the subscript $u$ will be used $\left(\Xi_{u}\right)$. Similarly, $\Xi_{d}$ will depict the distribution of the downwelling radiances. All the "apparent" optical properties (AOPs; sensu Preisendorfer 1961) depend on, and can be obtained from, the radiance distribution.

A paradox in marine optics is that the geometrical structure of the underwater light field is generally ignored when we measure and interpret underwater optical properties (e.g., diffuse attenuation or absorption and scattering), whereas it has a strong impact on many of these properties. Measuring $L(\Xi)$ has long been an elusive task because of technological limitations, whereas accurate knowledge of the changes of $L(\Xi)$ with depth would actually allow IOPs and AOPs to be derived consistently (e.g., Zaneveld 1989). The $L\left(\Xi_{u}\right)$ is partially known in open ocean waters (Voss et al. 2007; Voss and Morel 2005; Morel et al. 1995) and sparsely documented in coastal turbid waters (Gleason et al. 2012). Measurements of $L\left(\Xi_{d}\right)$ have been carried out only recently with present developments (see also Lewis et al. 2011). They had not been measured since early attempts in the 1960s and 1970s (e.g., Tyler 1960; see below for a more complete historical background).

Pushing back this frontier is not only important for the fundamental knowledge of how light interacts with matter in the ocean. It also has multiple implications and applications for better field measurements of optical properties, and better interpretation and use of these properties as they are measured from ocean color satellite remote sensing observations. Optical properties are of tremendous importance for many other domains of oceanography and for marine operations (e.g., heating rate of the upper ocean, biogeochemistry and carbon cycle, water transparency, coastal zones monitoring, assimilation into global coupled physical-biological models, and underwater imaging). Improving their quality and consistency is therefore an important goal.

Here we present a new miniaturized underwater radiance camera, called the CE600, which has been specifically developed to allow multispectral, high-radiometric accuracy $L(\Xi)$ measurements. The purpose is to show how this instrument has been conceived, built, characterized, and calibrated, and to present preliminary results from field deployments. These examples illustrate the performance of this instrumentation and how the data it provides could fundamentally renew the way of deriving optical properties from profile measurements of $L(\Xi)$ in the water column. Important features that are emphasized in comparison to previously developed instruments (Voss and Chapin 2005; Lewis et al. 2011) include 1) miniaturization, which allows the minimization of the unavoidable self-shading when measuring the upwelling radiances; 2 ) a specifically designed fore optics with very high angular resolution and low attenuation; 3 ) the use of a detector array whose characteristics allow up- and downward hemispheres to be simultaneously measured; and 4) absolute calibration that is often neglected for such radiance camera systems.

\section{Historical background}

Although the radiance distributions and their transformation with increasing depth are the most fundamental properties in marine optics, measurements have not been performed in a systematic manner. Yet, an internally baffled tube (often called a Gershun tube), with an aperture limiting the acceptance angle and isolating a $d \Omega$ solid angle in a given direction at one end and at the other end a detector, provides a simple means of measuring radiance as defined above. The first problem when aiming to describe the entire radiance field is the orientation of such a radiance meter in polar and azimuth angles in a moving environment. The second problem is that the radiance distribution, which depends on the illumination conditions 

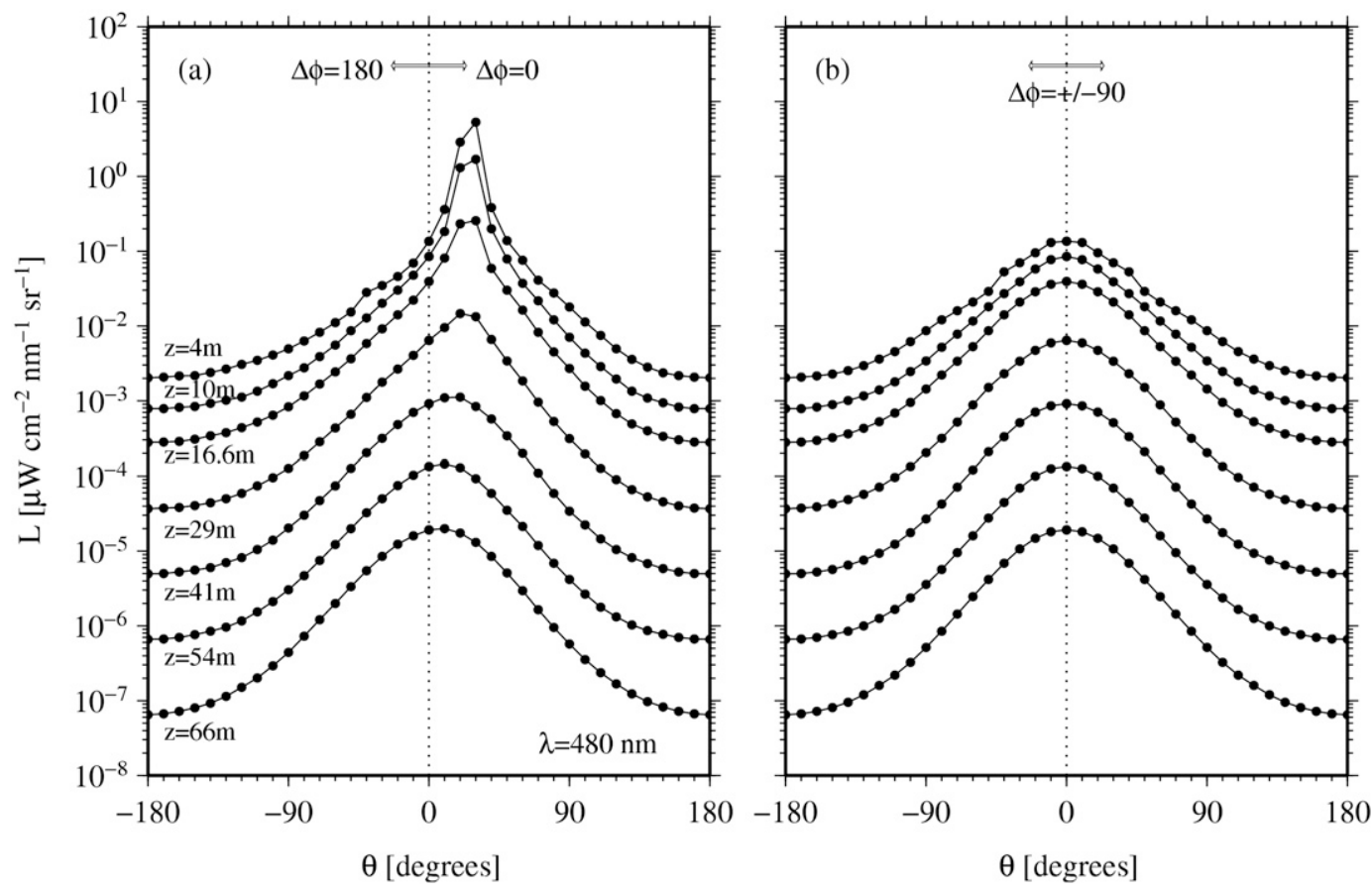

FIG. 1. Radiance distributions in the (a) principal and (b) perpendicular planes as measured by Tyler (1960) in Lake Pend Oreille for the depths indicated and $\lambda=480 \mathrm{~nm}$.

above the surface and the fluctuating state of the air-water interface (capillary waves in particular), must be measured in a short time. In spite of these obvious experimental difficulties, a few determinations were made before World War II (Jerlov and Liljequist 1938; Pettersson 1938). Historical accounts are given in Jerlov (1976) and in Hojerslev (1989) (see also Maffione 2001).

Several instruments using a Gershun tube and various mechanisms to control the orientation of the tube were developed in the 1950s-70s. Jerlov and Fukuda (1960) made measurements down to $30 \mathrm{~m}$ in a vertical plane with a fixed azimuth orientation by guiding the instrument along a taut wire anchored in Gullmar Fjord. Later, an instrument with four measuring tubes aiming at different polar angles and rotated around a vertical axis thanks to a propeller was operated in the Sargasso Sea by Lundgren and Højerslev (1971); determinations of the upward and downward radiance distribution were made at three wavelengths $(\lambda=425,475$, and $525 \mathrm{~nm})$ and down to $400 \mathrm{~m}$ (at $475 \mathrm{~nm}$ ). Sasaki et al. (1958) built an instrument using a photomultiplier tube, and remotely controlled it to measure the horizontal angular distribution of underwater radiance. Then (in 1961), the same group developed a sophisticated radiance meter (Sasaki et al. 1962) with two heads, motors, a magnetic compass, and a rudder, capable of measuring the submarine radiance distribution. The progressive modification of $L(\Xi)$ with increasing depth was described and the trend toward a diffuse light regime (or "asymptotic radiance" distribution) was corroborated by these early measurements. A set of $L(\Xi)$ measurements was collected in the Mediterranean Sea in 1971 by Norwegian oceanographers aboard the R/V Helland-Hansen. These data have been recently reanalyzed by Aas and Højerslev (1999) and Adams et al. (2002), who also give a historical account of underwater radiance measurements.

Actually, the first systematic and comprehensive radiance dataset was obtained in Lake Pend Oreille, Idaho, by Tyler (1960). This set includes complete radiance distributions (at 10 azimuth angles from $0^{\circ}$ to $180^{\circ}$, and at 19 zenith angles from $0^{\circ}$ to $180^{\circ}$ ), which were determined at seven depths $(4.2-66.1 \mathrm{~m})$ with a clear sunny sky and at five depths $(6.1-55 \mathrm{~m})$ under an overcast sky. This set has often been used to validate radiative transfer computations (see Fig. 1).

A completely different type of collector is the fish-eye lens with a $180^{\circ}$ field of view. Associated with a photographic camera, such a device allows the complete radiance field within a half-space to be instantaneously captured. The instrument developed by Smith et al. (1970) contained two cameras of this type placed back to back, so that the full radiance distribution is measured in two images; the film exposure was remotely controlled. The quantitative values of the radiance were obtained (in relative units) from a microdensitometer scan of the negative. Radiance distributions to about seven optical 
depths were obtained under a variety of environmental conditions and water types (Smith 1974). The problem related to the orientation and the needed quickness of the measurements is solved with such equipment. The simultaneous acquisition and the storage of large volumes of data are also possible with the photographic approach; its drawback, however, is the rather poor performance of the photographic radiometry. A more basic application of this concept was actually attempted by Ivanoff and Moreuil (1963), who reported few measurements of the downwelling radiances at small scattering angles around the direct sunbeam. These measurements were taken with a commercial camera and recorded on negatives.

In terms of instrumentation, the step beyond was represented by the electro-optic radiance camera system (RADS) developed by Voss (1989a). This system is also based on fish-eye lenses and on two electro-optic chargeinjection device cameras $(260 \times 253$ pixels $)$; the interference filter changer is controlled from the surface. Improved versions of this instrument (RAD-II) were subsequently developed by using sensors with enhanced sensitivity and by decreasing the overall size, thus decreasing the instrument self-shading (Voss and Chapin 1992). The third version of this camera system (NuRADS) was particularly dedicated to the measurement of the upwelling radiance distribution just beneath the sea surface (Voss and Chapin 2005). Based on three NuRADS instruments in which linear polarizers have been installed, another system was developed to determine the polarization state of the upward light field just beneath the sea surface (PolRADS instrument; Voss and Souaidia 2010). The concept has further evolved and led to additional designs and measurements (Bhandari et al. 2011a,b). The concept of a free-fall profiling system using two cameras head-to-tail configuration was actually also developed by Lewis et al. (2011) in parallel to our work. Their camera uses a commercial fish-eye lens, a complementary metal oxide semiconductor (CMOS) array, and is monospectral (one band centered at $555 \mathrm{~nm}$ ). The sole alternative design to our knowledge was proposed by Haltrin et al. (1997), using a scanning radiance meter.

Though only a few radiance field determinations were made before the introduction of the fish-eye as collector, during this period considerable theoretical work was carried out to understand and predict the in-water light field. This work required the development of analytical techniques to numerically solve the RTE [see, e.g., review in Jerlov (1976) and in Zaneveld (1974)]. One recurrent motivation was the presumed existence of an "asymptotic radiance distribution" (or diffuse deep regime), which would set up with progressively increasing depth (Prieur and Morel 1971; Zaneveld and Pak 1972). Both experimental and theoretical efforts to detect and characterize this regime were attempted. Actually, elaborate numerical methods for the radiation transfer problem were only feasible when electronic computers became available. The simulation of the photons' fate and transport via Monte Carlo methods was a powerful approach (Plass and Kattawar 1969; Gordon et al. 1975; Kirk 1981); other numerical methods also proved to be efficient and a comparison of seven numerical models for producing the full underwater light fields demonstrated that these models are able to provide an accurate solution of the RTE for any optical oceanographic problem (Mobley et al. 1993).

Motivated by the development of the ocean color remote sensing technique, a computational effort was made to interpret quantitatively the upward radiant flux as it emerged from the ocean surface (e.g., Gordon et al. 1975; Gordon and McCluney 1975; Morel and Prieur $1977)$ and to relate the irradiance reflectance $(R$; an AOP) to the IOPs. Thereafter, as an important result of theoretical calculations, it was realized that the bidirectional character of the oceanic reflectance is a phenomenon to be considered in the remote sensing problem (Morel and Gentili 1991, 1993, 1996). Were the upward radiance field isotropic $\left(L_{u}\right.$ constant whatever $\theta$ and $\phi$ are), Eq. (6) would lead to $E_{u}=\pi L_{u}$. But that is not the case; $L_{u}$ depends on $\theta$ and $\phi$, so that $\pi$ is replaced by a bidirectional function, $Q(\theta, \phi)$. The first validation of these predictions was obtained by comparing them with experimental determinations of the upward radiance field made with the RADS instrument (Morel et al. 1995); other comparisons were then obtained with the NuRads instrument (Voss and Morel 2005; Voss et al. 2007), all in oceanic Case-1 waters.

\section{Fundamental CE600 instrument design}

\section{a. Overall design}

Self-shading during measurement of the upwelling radiances is directly related to the size of the instrument. Miniaturization was accordingly one of the main drivers when designing the CE600 camera system. The entire optics and most of the electronics are contained in a cylindrical housing of $96-\mathrm{mm}$ diameter by $130-\mathrm{mm}$ length (Fig. 2), which is a reduction in size by a factor of about 2.5 as compared to the NuRADS system (a factor of $\sim 15$ in volume). The flat part extending on the back was needed to host a loop of the fiber optics that connects the output of the detector array (see section 3d) to the optical modem. The housing is made of anodized aluminum and is rated to reach a $200-\mathrm{m}$ depth. The hemispherical glass 


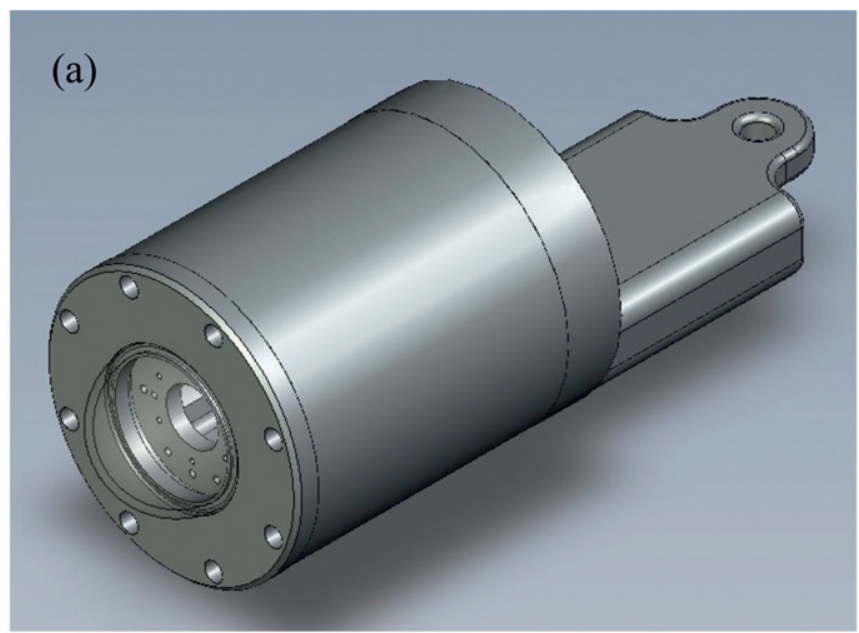

(b)

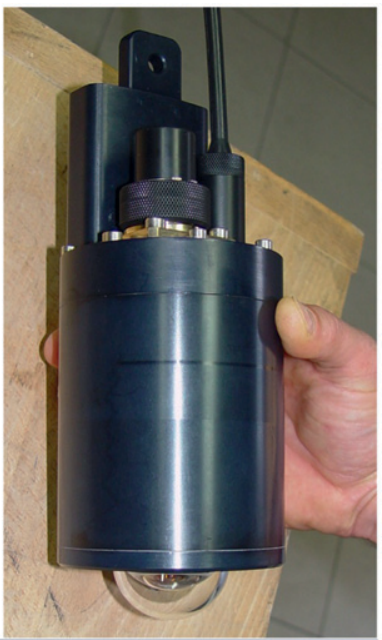

(c)

Filter wheel CMOS detector array

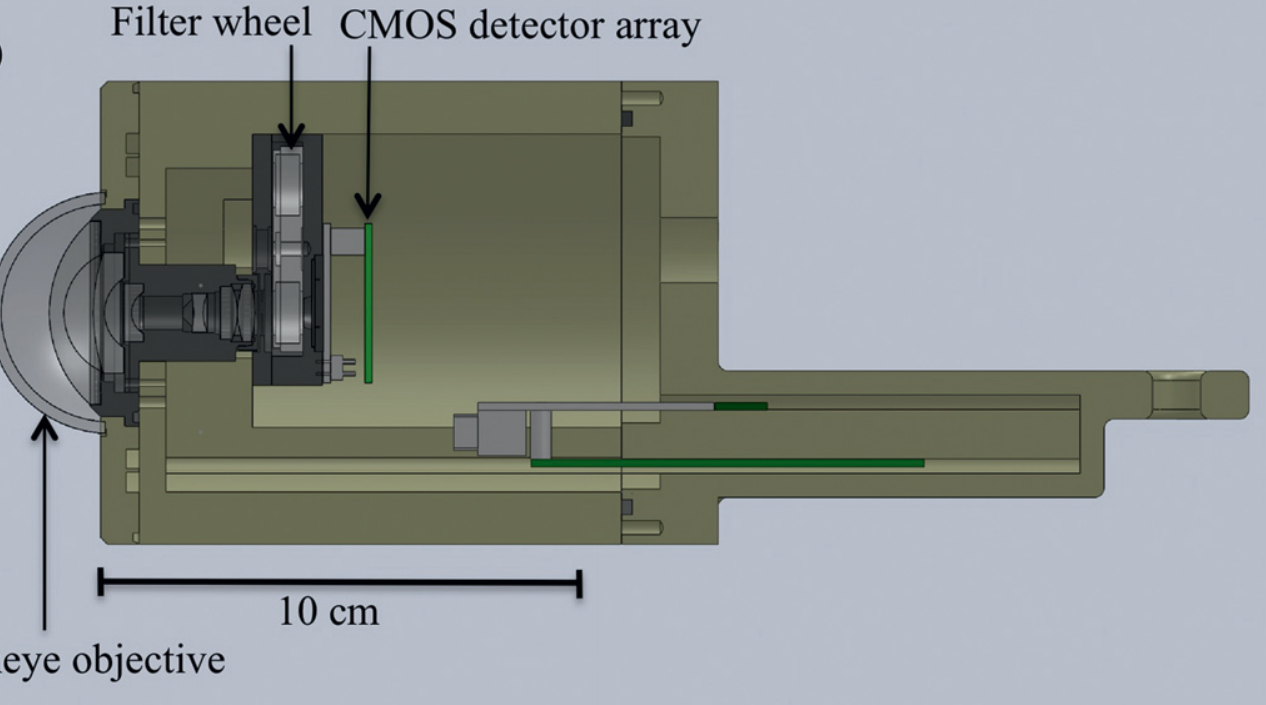

FIG. 2. (a) 3D external view of the CE600 camera system, (b) picture showing the scale, and (c) section showing how the main elements are organized internally.

dome is glued in a circular slot engraved on the upper faceplate. A fiber-optic connector and a wet pluggable 3-pin electrical connector occupy most of the back plate. Building such a small instrument was possible by using fiber optics to transmit the full data frames directly to the control computer onboard the ship. The only equipment and electronics inside the instrument container are the controller of the detector array, the optical modem, and ancillary sensors for pressure, internal temperature and humidity, two-axis tilt, and compass (see section 3e).

\section{b. Fore optics (fish-eye objective)}

Commercial lenses capturing a $2 \pi$-sr field of view have a large front part and a complex structure required for the correction of optical aberrations, including the chromatic aberration. This complex structure leads to high costs. Moreover, the actual field of view is often limited by the size of the detector, which is slightly smaller than the image. These nonoptimal characteristics led to the development of a lens specifically adapted to measure radiances in 20-40-nm-wide spectral bands (Figs. 3, 4 ). This objective does not have to correct for chromatic aberrations, which means that the size of the image and the angular resolution slightly depend on the wavelength. It has been the subject of a patent (No. 2838528; under the name Objectif de prises de vues a champ de $180^{\circ}$ et de structure simplifiée).

The objective is made of a front part and a rear part. The front part, which is usually quite complex for classical objectives (about 10 elements), is here simplified to 


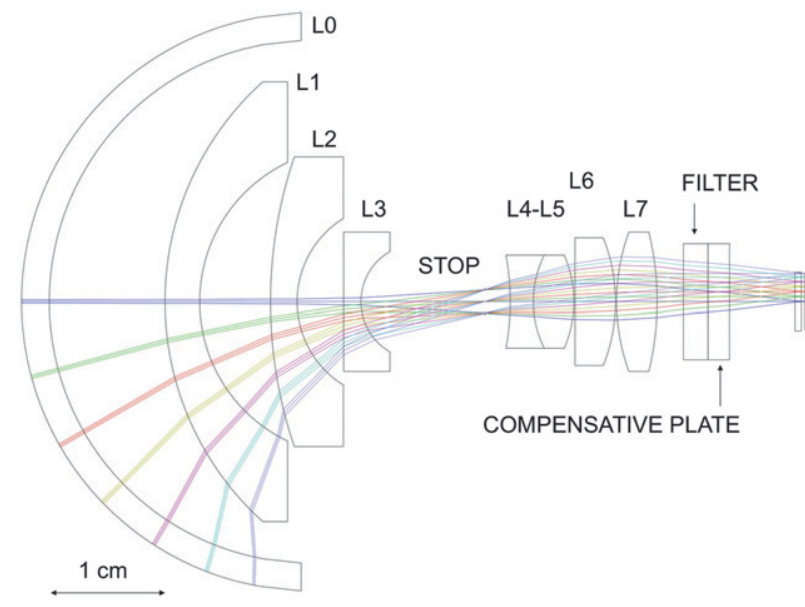

FIG. 3. Design of the fish-eye lens system (see Table 1 for the characteristics of each element identified here from L0 to L7).

4 elements (Fig. 3), thus reducing considerably the diameter of the optics. Another way to reduce the overall size of the system is to reduce the effective focal length of the system. This size reduction has to go with a high resolution to get enough information on the small image size. By neglecting chromatic aberrations, it is quite easy to get good quality in the entire $180^{\circ}$ field of view. The front part includes two divergent meniscuses with their faces toward the incoming light. A diverging lens is added to relax tolerances on both meniscuses. The rear part includes three converging elements. Two are simple converging lenses, and the third one is a cemented doublet with a divergent surface. To achieve aplanetism in the image plane, the focal lengths of the two parts must be of opposite sign and differ by less than $20 \%$. The objective has an effective focal length of $1.2 \mathrm{~mm}$ and works at $\mathrm{F} / 4.1$. The image diameter for the field at $180^{\circ}$ is $4.8 \mathrm{~mm}$ at $494 \mathrm{~nm}$. The optical system can capture incident rays from normal incidence up to slightly more than $90^{\circ}\left(\sim 92^{\circ}\right)$. Collecting rays from directions slightly greater than $90^{\circ}$ allows for joining the measurements of the upward and downward hemispheres when using two cameras in a head-to-tail configuration. A protective hemispherical glass dome covers the optics and represents the first interface with the marine environment. All lenses have been specifically antireflection coated to minimize reflections and stray light (see Table 1 for more detail).

To achieve a good wavelength selection and to reduce the out-of-band background noise, bandpass optical filters are used with an incoming beam angle of incidence on the filter lower than $12^{\circ}$. For this reason, the diaphragm of the lens is placed at the focal object of the first optical part and the filters are placed in the second part. Thus, for each angle of view, the principal ray

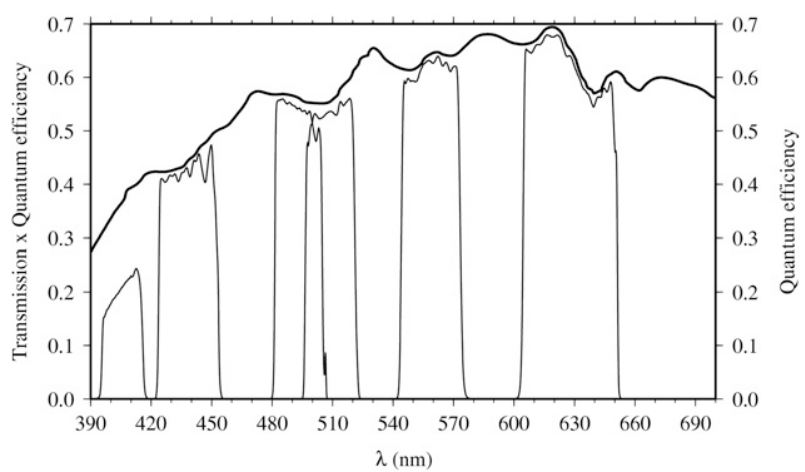

FIG. 4. Spectral response of the six filters of the CE600 camera system times the QE of the CMOS detector (thin curves). The QE is separately shown as the bold curve.

passes through the center of the diaphragm and goes out parallel to the optical axis toward the optical filter. The marginal ray that passes on the edge of the diaphragm determines the maximum angle of incidence on the filters. The diaphragm determines the solid angle subtended by the illuminated pixel. Its diameter is adjusted so as to limit the maximum angle of incidence at $7^{\circ}$.

\section{c. Spectral filters and filter wheel}

The six spectral filters were selected so as to satisfy three requirements: optical transmission must be as high as possible (typically $T>80 \%$ ) to allow low radiances to be measured. Second, because of the high background radiance level when pointing upward, the out-of-band optical density (OD) of the filters must be as high as possible too (typically OD $>6$ from 300 to $1.2 \mu \mathrm{m}$ ). Finally, the filters must resist direct sun exposure. Hardcoated filters using ion-beam sputtering deposition methods are the only ones to satisfy these requirements. Semrock bandpass-type filters were selected. Their central wavelengths and bandwidth are given in Table 2, and their detailed spectral shapes are shown in Fig. 4. Their large bandwidths were chosen so as to maximize the signal-to-noise characteristics. These filters are mounted in a PC-controlled filter wheel driven by a step motor. This wheel is also used in a half-position (i.e., between two filters) for the measurement of the detectors' dark current. Chromatic aberrations are such that they introduce a "defocusing" of the image, that is, the back focal length varies slightly with the wavelength. To overcome this without using moving parts, compensative wavelength-specific glass plates are added on top of the filters (Table 2). Moving from one filter to the next one requires about $188 \mathrm{~ms}$. The six spectral bands are therefore acquired within about $2 \mathrm{~s}$ for an average integration time of $200 \mathrm{~ms}$, which is typical of what is needed in the field (see section 5). 
TABLE 1. Characteristics of the different elements of the fish-eye objective (see Fig. 3). The negative radii in the second column mean that the face is curved toward the rear.

\begin{tabular}{|c|c|c|c|c|c|}
\hline Objective element & $\begin{array}{l}\text { Outer and inner } \\
\text { radii }(\mathrm{mm})\end{array}$ & Thickness (mm) & $\begin{array}{l}\text { Distance to next } \\
\text { element* }(\mathrm{mm})\end{array}$ & Glass type & $\begin{array}{l}\text { Outer and inner } \\
\text { diameters }(\mathrm{mm})\end{array}$ \\
\hline \multirow[t]{2}{*}{ L0 } & 25.00 & 2.40 & 10.00 & B270 & 49.97 \\
\hline & 22.60 & & & & 45.17 \\
\hline \multirow[t]{2}{*}{ L1 } & 25.59 & 3.00 & 6.12 & SF6 & 38.00 \\
\hline & 13.32 & & & & 24.06 \\
\hline \multirow[t]{2}{*}{ L2 } & 39.13 & 2.30 & 4.02 & N-SF8 & 25.00 \\
\hline & 8.45 & & & & 14.37 \\
\hline \multirow[t]{2}{*}{ L3 } & & 1.50 & 10.70 & N-SF8 & 12.00 \\
\hline & 4.97 & & & & 8.63 \\
\hline Stop & & 2.20 & & & 2.00 \\
\hline L4 & -22.00 & 2.00 & & N-SF57 & 8.00 \\
\hline \multirow[t]{2}{*}{ L5 } & 9.00 & 3.50 & 0.10 & N-SF64 & 8.00 \\
\hline & -10.00 & & & & 8.00 \\
\hline \multirow[t]{2}{*}{ L6 } & & 3.50 & 0.10 & N-BAK4 & 11.00 \\
\hline & -14.77 & & & & 11.00 \\
\hline \multirow[t]{2}{*}{ L7 } & 17.59 & 3.50 & 2.27 & N-BAK4 & 12.00 \\
\hline & -25.37 & & & & 12.00 \\
\hline Filter & & 2.12 & & BOROFLOAT & 10.00 \\
\hline \multirow[t]{2}{*}{ Plate } & & 1.87 & 5.65 & N-BK7 & 10.00 \\
\hline & & & & & 10.00 \\
\hline \multirow[t]{2}{*}{ Silica** } & & 0.55 & 0.29 & D263T & 5.02 \\
\hline & & & & & 4.93 \\
\hline CMOS array & & N/A & & & 4.88 \\
\hline
\end{tabular}

* Distance from the inner surface of the element in question to the outer surface of the next element, taken along the optical axis.

** This is a silica protection window for the CMOS sensor.

\section{d. CMOS detector}

The selection of the detector array was essentially driven by the need to get high-radiometric accuracy measurements of low in-water radiances (e.g., close to the $1 \%$ light level or at the surface with a low sun elevation), as well as high radiances expected when pointing upward just beneath the sea surface (including the image of the sun or, at least, of the rays forwardly scattered at small angles off the direct sun). Typical values for several spectral domains and conditions are given in Table 3 [from radiative transfer computations, as in Morel and Gentili (2004)]. They were used as constraints for the selection of the detector array. Cost and availability were two additional constraints.
The choice was eventually for a complementary metal oxide semiconductor detector array. Because CMOSs convert the charge to voltage directly in the pixels and because they do not use shift registers, they have inherent antiblooming and antismearing characteristics. Incoming photons are converted to electrons at each pixel. During the integration time, all the generated electrons are stored on each photodiode's capacitor. At the end of each integration time, the analog signal is transferred to the column bus, where the column buffer amplifies the photo-generated signal voltage in conjunction with the pixel circuitry. A charge-coupled device (CCD) array would, in principle, provide higher sensitivity and probably better linearity at high radiances, which actually are unexpected for marine optics

TABLE 2. Characteristics of the six spectral filters and compensating glass plates used in the CE600 radiance camera.

\begin{tabular}{|c|c|c|c|c|c|c|}
\hline \multicolumn{5}{|c|}{ Filters } & \multicolumn{2}{|c|}{ Glass plates } \\
\hline No. & $\begin{array}{c}\text { Central } \\
\text { wavelength }(\mathrm{nm})\end{array}$ & $\begin{array}{l}\text { FWHM (full width } \\
\text { at half maximum; nm) }\end{array}$ & $\begin{array}{c}T_{\text {avg }}(\operatorname{avg} \\
\text { transmittance; \% })\end{array}$ & Thickness (mm) & $\begin{array}{l}\text { Type (nomenclature } \\
\text { of Schott glasses) }\end{array}$ & Thickness (mm) \\
\hline 1 & 406 & 15 & 85 & 2.10 & $\mathrm{SF} 2$ & 1.60 \\
\hline 2 & 438 & 24 & 93 & 3.60 & $\mathrm{SF} 2$ & 1.00 \\
\hline 3 & 494 & 20 & 93 & 3.60 & Silica & 0.93 \\
\hline 4 & 510 & 10 & 93 & 2.00 & N-BK7 & 2.05 \\
\hline 5 & 560 & 25 & 93 & 1.90 & $\mathrm{~N}-\mathrm{BK} 7$ & 1.87 \\
\hline 6 & 628 & 40 & 93 & 3.60 & Silica & - \\
\hline
\end{tabular}


TABLE 3. Typical radiance values $\left(\mathrm{W} \mathrm{m}^{-2} \mathrm{~nm}^{-1} \mathrm{sr}^{-1}\right)$ in the underwater environment, for the depths indicated $\left(0^{-}\right.$means just beneath the sea surface; $Z_{e}$ indicates the $1 \%$ light level). The radiative transfer computations that led to these results were performed using the Hydrolight code (Mobley 1994) for various solar elevations (from $15^{\circ}$ to $75^{\circ}$ ) and for chlorophyll concentrations from 0.03 to $10 \mathrm{mg} \mathrm{m}^{-3}$. Raman scattering was included.

\begin{tabular}{|c|c|c|c|c|c|c|}
\hline & \multicolumn{2}{|c|}{$0^{-}$} & \multicolumn{2}{|c|}{$1 / 4 Z_{e}$} & \multicolumn{2}{|c|}{$3 / 4 Z_{e}$} \\
\hline & Min & Max & Min & Max & Min & Max \\
\hline \multicolumn{7}{|c|}{ Upwelling radiances } \\
\hline $412 \mathrm{~nm}$ & $1 \times 10^{-3}$ & $1 \times 10^{-1}$ & $2 \times 10^{-4}$ & $5 \times 10^{-2}$ & $5 \times 10^{-5}$ & $2 \times 10^{-2}$ \\
\hline $560 \mathrm{~nm}$ & $5 \times 10^{-4}$ & $5 \times 10^{-2}$ & $1 \times 10^{-4}$ & $5 \times 10^{-2}$ & $5 \times 10^{-5}$ & $2 \times 10^{-2}$ \\
\hline $660 \mathrm{~nm}$ & $2 \times 10^{-4}$ & $2 \times 10^{-2}$ & $5 \times 10^{-5}$ & $5 \times 10^{-3}$ & $5 \times 10^{-5}$ & $5 \times 10^{-5}$ \\
\hline \multicolumn{7}{|c|}{ Downwelling radiances (excluding the direct sunbeam) } \\
\hline $412 \mathrm{~nm}$ & $1 \times 10^{-3}$ & 1 & $1 \times 10^{-3}$ & 1 & $1 \times 10^{-4}$ & 1 \\
\hline $560 \mathrm{~nm}$ & $5 \times 10^{-3}$ & 1 & $2 \times 10^{-4}$ & 1 & $5 \times 10^{-5}$ & $5 \times 10^{-1}$ \\
\hline $660 \mathrm{~nm}$ & $5 \times 10^{-4}$ & 1 & $5 \times 10^{-5}$ & 1 & $5 \times 10^{-5}$ & $5 \times 10^{-3}$ \\
\hline
\end{tabular}

applications. Such CCD detectors have, however, the weakness of leaking charges to adjacent pixels when the CCD register overflows, causing unwanted streaks in the image (blooming). The selected array is the ProCamHD 3560 image sensor manufactured by Altasens (Westlake Village, California), which is specifically optimized to provide superior performance against competing high-definition CCDs in most respects including random noise, antiblooming, smear, power consumption, and video rate (main characteristics in Table 4). The quantum efficiency (QE) of this CMOS array is close or superior to $60 \%$ for wavelengths greater than $\sim 470 \mathrm{~nm}$ and decreases to $\sim 40 \%$ around $400 \mathrm{~nm}$ (Fig. 4). Integration times as short as $19 \mu$ s are possible and can be increased up to $1.24 \mathrm{~s}$ by steps of $19 \mu \mathrm{s}$. We used this CMOS as already integrated into the reference SI1920HD-M-S-T digital camera manufactured by Silicon Imaging, Inc. (Niskayuna, New York).

\section{e. Data transmission and ancillary sensors}

An in-house electronic board designed and produced by CIMEL controls the different camera elements, in particular the filter wheel. It also receives the signal from ancillary sensors, which include a pressure sensor [Honeywell Precision Pressure Transducer-Ruggedized (PPTR); typical accuracy $\pm 0.1 \%]$, a three-axis, tilt-compensated compass (Honeywell HMR 3300; tilt accuracy $1^{\circ}$ RMS between $0^{\circ}$ and $\pm 30^{\circ}$ ), a digital humidity and temperature sensor (Sensirion SHT $15 / 75$; temperature accuracy $\sim 0.5^{\circ} \mathrm{C}$; relative humidity accuracy $0.03 \%$ ), and internal voltage sensors.

The outputs of the CMOS are sent to an Ethernet module (Pleora Technologies, iPort FB-1000CL) at $1 \mathrm{~Gb} \mathrm{~s}^{-1}$ (CameraLink format). This Ethernet module controls the data flux through the optical fiber (up to $200 \mathrm{~m}$ for a multimode fiber, and up to $1000 \mathrm{~m}$ with a single mode fiber), in conjunction with another Ethernet board that controls the CE600 on the computer side. The acquisition software allows for controlling the acquisition parameters (integration time, gain, sequence of filters) and provides real-time measures of pressure, temperature, and other internal parameters.

TABLE 4. Main characteristics of the ProCamHD 3560 CMOS array manufactured by Altasens.

\begin{tabular}{ll}
\hline \hline Technology node & United Microelectronics Corporation (UMC) $0.25-\mu \mathrm{m}$ technology for CMOS imaging sensors \\
Resolution & $1936 \times 1090$ visible pixels, 4 black rows, 72 black columns \\
Optical format & $2 / 3$ inch [high-definition TV (HDTV) 1080p or extended sensitivity 720p] \\
Pixel area & $5 \mu \mathrm{m} \times 5 \mu \mathrm{m}$ \\
Pixel rate & Nominal $150 \mathrm{or} 74.25 \mathrm{MHz}(274 \mathrm{M}$ standard from the Society of Motion Picture and Television Engineers) \\
Image capture & Electronic Focal Plane Rolling Shutter \\
Supply voltage & $3.3 \mathrm{~V}$ analog and $2.5 \mathrm{~V}$ digital \\
Power consumption & $<650 \mathrm{~mW}$ \\
Gain & Two analog gain stages with gain ranges of $0 \rightarrow+24 \mathrm{~dB}$ and $-6 \rightarrow+18 \mathrm{~dB}$, respectively, adjustable \\
& in 3-dB steps \\
& One digital gain stage (multiplier) with gain range from -24 to $+72 \mathrm{~dB}$, adjustable in 0.006- and 6-dB steps \\
Random noise (RMS) & 5 least significant bits \\
Saturation capacity & $>50$ ke-(kiloelectrons) \\
Dynamic range & $68 \mathrm{~dB}(>11$ bits) \\
\hline
\end{tabular}



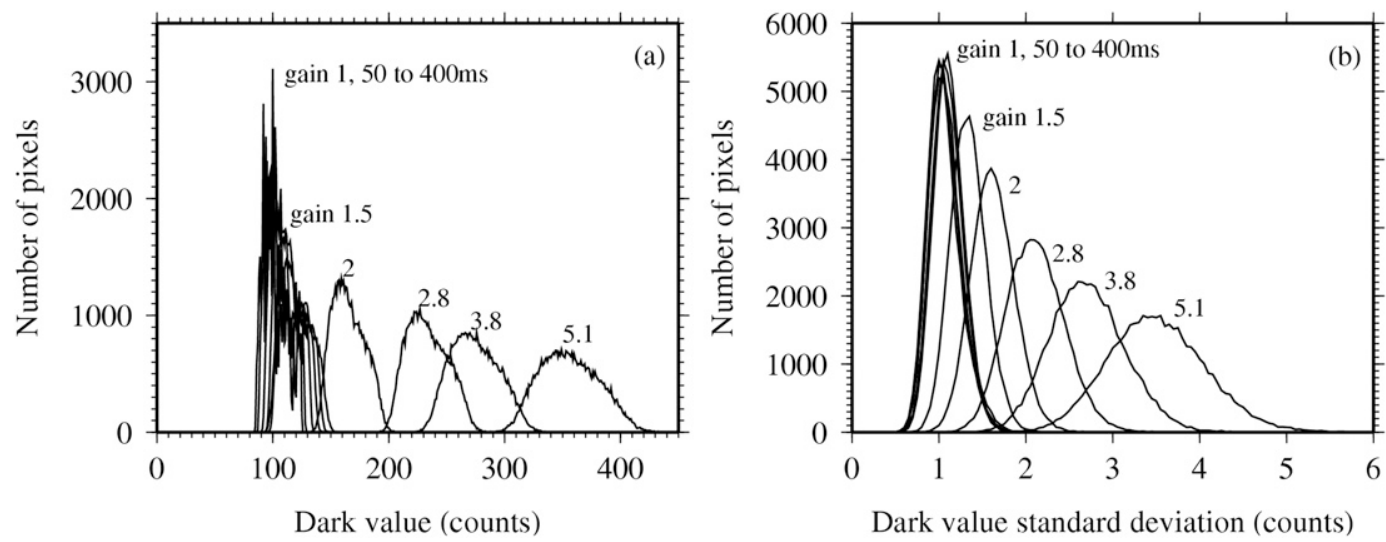

FIG. 5. (a) Histograms of the dark current measurements over the CMOS area corresponding to view angles $<92^{\circ}$, as accumulated over 25 successive image acquisitions. The superimposed histograms on the left-hand side are for an electronic gain of 1 and integration times increasing from 50 to $400 \mathrm{~ms}$, as indicated. Other histograms are for an integration time of $400 \mathrm{~ms}$ and the electronic gains indicated. (b) Histograms of the standard deviation of the CMOS dark current on every pixel of the CMOS area used in (a).

\section{Instrument characterization and calibration}

The methods for optical and radiometric characterization of the CE600 camera are largely inspired from procedures previously published for similar instruments (Voss and Zibordi 1989; Voss and Chapin 2005). Full details of these procedures are beyond the scope of this paper. Only their main specific aspects are presented and emphasis is put on the results of the different characterization steps.

Results presented will be (unless otherwise indicated) for a data averaging over $4 \times 4$ pixels. This averaging aims at 1) improving the signal-to-noise ratio, 2) removing a periodical modulation of the image (see below), and 3) reducing the data volume. The image modulation is due to the construction of the CMOS sensor. This sensor uses four independent analogic circuitries to read the matrix's columns by groups of four to increase the reading speed. These four independent circuitries do not have the same gain, which produces a modulation of the signal with a periodicity of four columns. The angular resolution is still excellent after the $4 \times 4$ averaging (see section $4 b$ ).

\section{a. Dark noise, SNR, dynamic range, and linearity of the CMOS detector array}

The experimental setup to determine the dark noise, signal-to-noise ratio (SNR), and dynamic range of the camera consisted of placing the camera in front of a Spectralon plaque illuminated by a power-stabilized $1000 \mathrm{~W}$ FEL halogen lamp (i.e., a typical setup for absolute calibration; see further details below). Several measurement protocols were then followed.
To characterize the dark noise, which depends on the gain and integration time and varies from pixel to pixel, series of sequential images (25) have been taken for various integration times and gains, with a cap covering the entire camera optics. The results show that the dark count noise increases with gains (Fig. 5a). For instance, the histogram of dark counts is centered on 120 with a width of 50 when the gain is minimum, while it is centered on 350 with a width of 100 when the gain is equal to 5.1. The standard deviation of the dark counts is small (Fig. 5b, see caption for details), from 1 for the smallest gain to 3.5 on average for the highest one. When the gain is 1 , the dark counts and their standard deviation do not vary significantly with integration time. That means that the dark noise is mostly due to reading noise, with the consequence that the variation of the dark noise with temperature is weak (experimentally verified; results not shown).

The SNRs were also determined with the setup described above. The radiances measured in this configuration (Table 5) are in the range of what is expected underwater (cf. Table 3), even on the low side at $406 \mathrm{~nm}$. Values in Table 5 are those reached just before saturation (i.e., at maximum SNR). They are obtained by increasing the integration time first (in the limit of $400 \mathrm{~ms}$ ) and, if not enough to approach saturation, by increasing the gains. This is the strategy used in the field. Twentyfive successive images plus a dark image have been acquired for each wavelength. The noise is computed as the average over the 25 images of the standard deviation of each pixel included in the image of the plaque. The resulting SNR varies from 154 at $406 \mathrm{~nm}$ to more than 800 at $628 \mathrm{~nm}$. 
TABLE 5. Noise characteristics of the CMOS sensor (see section 4a).

\begin{tabular}{|c|c|c|c|c|c|c|}
\hline Wavelength (nm) & 406 & 438 & 494 & 510 & 560 & 628 \\
\hline Radiance $\left(\mathrm{W} \mathrm{m} \mathrm{mm}^{-1} \mathrm{sr}^{-1}\right.$ ) & $4.7 \times 10^{-4}$ & $7.5 \times 10^{-4}$ & $1.4 \times 10^{-3}$ & $1.6 \times 10^{-3}$ & $2.3 \times 10^{-3}$ & $3.2 \times 10^{-3}$ \\
\hline Gain & 5.1 & 2.8 & 2 & 2.8 & 1 & 1 \\
\hline Integration time (ms) & 400 & 400 & 370 & 360 & 360 & 160 \\
\hline $\begin{array}{l}\text { Standard deviation } \\
\qquad\left(\mathrm{W} \mathrm{m} \mathrm{mm}^{-2} \mathrm{sr}^{-1}\right)\end{array}$ & $3.03 \times 10^{-6}$ & $2.04 \times 10^{-6}$ & $2.67 \times 10^{-6}$ & $4.07 \times 10^{-6}$ & $2.67 \times 10^{-6}$ & $3.73 \times 10^{-6}$ \\
\hline Signal-to-noise ratio & 154 & 370 & 529 & 393 & 848 & 848 \\
\hline Dark counts & 365 & 240 & 167 & 234 & 121 & 109 \\
\hline Useful dynamics (counts) & 3672 & 3816 & 3897 & 3822 & 3951 & 3963 \\
\hline
\end{tabular}

The corresponding dark counts, when subtracted from the maximum value of 4096 (12-bit digitization), provide the actual dynamics for a single image. It is large enough for measurements of upwelling radiances in any conditions. It is insufficient for measurements of downwelling radiances close to the surface. To overcome this limitation, it is possible to combine several images successively acquired with different integration times. When using the entire range of possible integration times and gains, the signal dynamic that can be explored with the CE600 is of 7 decades, which is large enough for marine applications.

The linearity of the CMOS detector was determined by placing it directly in front of the $1000 \mathrm{~W}$ FEL halogen lamp, without the fish-eye optics in between. The distance between the sensor and the lamp could be varied from $20 \mathrm{~cm}$ to $2 \mathrm{~m}$, leading to an order of 2 magnitude change in irradiance. Four experiments, with different current settings and using neutral density filters of varying transmission, were used to investigate linearity over 5 decades. Results showed an excellent linearity over this range. The average deviation from a perfect linearity, that is, from the radiance predicted from an inverse relationship with the square of the distance, is on average $0.5 \%$.

\section{b. Angular resolution and geometrical projection}

To determine its angular resolution, the CE600 camera is illuminated by a collimated beam, first parallel to the camera principal axis and then rotated by successive small angles. To produce a parallel and homogenous beam as large as the aperture of the CE600 objective, the flux from a halogen lamp is collimated using two lenses (focal lengths of 25 and $75 \mathrm{~mm}$ ), between which is a diaphragm of aperture size of $200 \mu \mathrm{m}$ (this arrangement theoretically produces a beam divergence of $0.075^{\circ}$ ). When the beam is parallel to the camera axis, the corresponding image is a single peak encompassing a few $(\sim 4)$ pixels at the center of the detector array (true pixels, not $4 \times 4$ averages). The angular resolution is determined as soon as the peaks resulting from the smallangle rotations are clearly separated. This procedure is repeated for all spectral bands because the size on the detector array of the image of the $2 \pi$ field of view varies with wavelength (with the focal length). It is also repeated for view angles from $0^{\circ}$ (camera axis) up to $90^{\circ}$. The angular resolution is close to half a degree in the nadir direction (camera principal axis) down to $2^{\circ}$ for a $90^{\circ}$ view angle.

The second part of the geometrical characterization consists of determining the view angle corresponding to each pixel of the CMOS detector array. A perfect fisheye objective would show a wavelength-independent linear relationship between the angle and the radial distance of a pixel from the center of the image. Design constraints and minor imperfections inherent to manufacturing necessitate the quantification of deviation from this theoretical law. A theoretical computation and an experimental characterization have been performed. The former was based on ray tracing, whereas the latter follows a similar experiment to the one described above for characterization of the angular resolution. Their results are in excellent agreement, that is, with a maximum difference of $1.1^{\circ}\left(0.43^{\circ}\right.$ on average). This difference is precisely what is accounted for by this calibration; that is, this is not an error introduced in the measurements.

\section{c. Roll-off of the fish-eye lens system}

The roll-off describes the attenuation along the entire optical path, from the outer glass dome to the detector, as well as the change in solid angle. It is experimentally determined by measuring the relative decrease of the measured radiance reflected by a spectralon plaque when the camera is rotated off axis, with respect to the value measured when the camera views the plaque under normal incidence. The results are shown in Fig. 6 for three spectral bands and four azimuthal planes equally spaced by $45^{\circ}$ from the others.

The transmission is maximal for $\lambda=494 \mathrm{~nm}$ (i.e., greater than 0.9 for view angles lower than $80^{\circ}$ and 0.85 when the angle reaches $\left.90^{\circ}\right)$. It is slightly lower for $\lambda=$ $406 \mathrm{~nm}\left(0.8\right.$ for a view angle of $\left.90^{\circ}\right)$ and only significantly degrades in the red. It decreases to 0.75 for a view angle of 


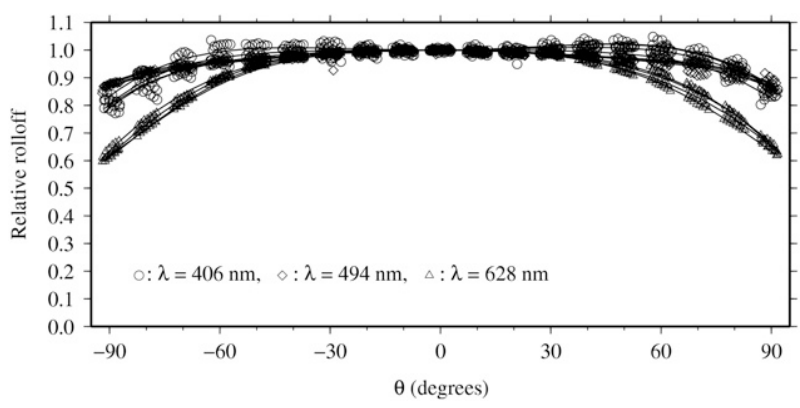

FIG. 6. Roll-off of the fish-eye lens objective for the three wavelengths indicated, as experimentally determined in four azimuthal planes (see text). The symbols are measurements and the lines are fit to these points.

$80^{\circ}$ for $\lambda=628 \mathrm{~nm}$. The four curves for the four azimuthal planes of the camera are not strictly identical, showing some heterogeneity of the overall camera response. A 2D function (zenith and azimuth angles) is therefore derived by fitting the roll-off values obtained along the four planes and is used to account for the system response.

The roll-off characterization is performed each time the instrument is used in the field, to account for possible changes due to the aging of optical parts (filters and lenses) or slight misalignments that may stem from hazardous instrument manipulations and from transport. The same procedure still has to be repeated with the camera immersed in a water tank to evaluate the change of the roll-off function between air and water.

\section{d. Polarization sensitivity}

To measure the total (unpolarized) radiance in the underwater environment where the radiance is actually partly polarized, the instrument should be insensitive to the polarization state (which is unknown when performing the measurements). This is actually infeasible because of design and manufacturing constraints, so it is necessary to quantify the unavoidable residual polarization sensitivity of the instrument. This is achieved by placing a linear polarizer between the camera and a spectralon plaque ( $45^{\circ}$ angle between both) illuminated by a $1000-\mathrm{W}$ FEL lamp. The camera and the polarizer are rotated so as to explore the residual polarization sensitivity for the full range of view angles and azimuthal directions of the camera. Such a procedure has been followed, and the results showed a residual sensitivity to polarization of about $1 \%$ for angles less than $60^{\circ}$. It reaches $5 \%$ for an incidence of $90^{\circ}$.

\section{e. Absolute radiometric calibration}

Absolute radiometric calibration was performed following the same protocol as for conventional radiance meters (e.g., Johnson et al. 1996). The camera views a calibrated spectralon plaque of known reflectance at an angle of $45^{\circ}$. The plaque is illuminated by a NISTcalibrated $1000 \mathrm{~W}$ FEL lamp. This setup provides an absolute calibration for the view angle corresponding to the camera principal axis, which is propagated for all other directions using the bidirectional roll-off function previously determined. However, this setup cannot generate high radiances typical of the downward radiances observed close to beneath the sea surface. Therefore, another absolute calibration was performed, by placing the camera at the entrance port of a calibrated integrating sphere. This second setup provided a calibration point for higher radiances (typically $0.9 \mathrm{~W} \mathrm{~m}^{-2} \mathrm{~nm}^{-1} \mathrm{sr}^{-1}$ at $\left.510 \mathrm{~nm}\right)$, which is used to calibrate $L\left(\Xi_{d}\right)$ measurements.

These in-air calibrations have to be complemented by the determination of the immersion factor. The immersion factor for a fish-eye camera describes the change in the camera response between in-water measurements and laboratory in-air calibration. This difference is due to the difference in refractive indexes between the first fish-eye element (the glass dome) and the medium (air or water). It is also due to the change of the apparent aperture size. Its value, denoted here as $C_{\mathrm{im}}$, the immersion coefficient, is determined from the law of conservation of optical étendue between two media (Meyzonnette and Lépine 2003):

$$
C_{\text {im }}=\left[1-\left(\frac{n_{g}-1}{n_{g}+1}\right)^{2} / 1-\left(\frac{n_{g}-n_{w}}{n_{g}+n_{w}}\right)^{2}\right] n_{w}^{2},
$$

where $n_{g}$ and $n_{w}$ are the refractive indexes of glass and water, respectively. With $n_{g}=1.52$ and $n_{w}=1.34$, the immersion coefficient is 1.72 . This value will have to be experimentally verified.

\section{Sample data from field experiments}

\section{a. Upward radiance distributions just beneath the sea surface}

The simplest deployment mode was designed to collect $L\left(\Xi_{u}\right)$ just beneath the surface (the " 0 " " level). The goal here is to study the distribution of the radiances emerging from the ocean (the water-leaving radiances; $\left.L_{w}\right)$, which are related to $L\left(\Xi_{u}\right)$ through the Fresnel and Snell laws. In this mode, one camera is equipped with a collar onto which three transparent Plexiglas tubes are attached $120^{\circ}$ apart from the others. These arms provide enough floatation for the lower half of the camera to remain below the water. The true depth of measurement is therefore about $10 \mathrm{~cm}$. The tubes' floatation was trimmed so as to provide some dumping 
of the movements due to surface waves. In the case of choppy seas, the tilt measurement is used to eliminate data collected for inclinations greater than some threshold (usually $5^{\circ}$ ). This floating camera is tethered to the ship via the fiber optic cable, and it is maintained at a sufficient distance from the ship to avoid seeing the ship hull in the field of view or being in the ship's shadow. The data acquisition software allows real-time visualization of the light field as recorded by the camera, so that integration times and electronic gains can be optimized in each given situation and for each spectral band, before data acquisition is triggered. Dark measurements are taken before and after each sequence of light measurements (using the appropriate position of the filter wheel). These measurements are occasionally complemented by dark current measurements taken with a cap covering the camera, and by reproducing the same sequence of gains and integration times as during the light measurements. Any sequence alternating varying numbers of dark and light measurements can actually be programmed as a function of the cast objectives.

Examples of such measurements are provided in Fig. 7. These sample data span a large range of conditions, from clear to overcast skies, from clear to very turbid waters, and for low and high sun elevations (see Table 6 for details). The four selected situations will be referred to as examples I to IV from the left to the right of the figure, where the six rows represent the six spectral bands. The corresponding water-leaving radiance spectra are displayed in Fig. 8a. They show that three orders of magnitude in radiance are explored using these four examples (and about two orders at a given wavelength). The remote sensing reflectance (RSR) spectra (Fig. 8b) clearly show two typical clear-water cases (Boussole and Malina station 235) and two turbid-water cases, one of which has very low reflectance in the blue and a maximum in the red (Malina station 696), and the other with enhanced reflectance across the entire visible domain and a maximum in the green $(560 \mathrm{~nm})$.

Under clear skies, the maximum radiance is always found on the sun side and view angles close to the horizon. Under an overcast sky (example IV) there is no longer direct sunlight. In such conditions the maximum radiance is observed near the horizon and for all azimuth angles. Under clear skies (examples I-III), the minimum radiance is located on the sun side. When the sky is overcast (example IV; with only diffuse sky light), the minimum is at nadir.

In examples I-III, deviations actually exist as compared to what the theory would predict (i.e., a minimum radiance in the principal plane). Such deviations probably result from various degrees of self-shading. For instance, the position of the minimum in example III actually matches that of one floatation arm. These arms are also likely responsible for the three bumps that distort the radiance distribution in this example. The shadows of these arms also clearly appear for $\lambda=628$ in example I. These perturbations, although very small, are likely to require a modification of the arms' design for future deployments.

The range of variations within one $L\left(\Xi_{u}\right)$ distribution can be as high as 28 (example II; $\lambda=628 \mathrm{~nm}$ ). It is generally closer to an average of $\sim 3$ in the blue and green bands for clear waters. It is composed between 1.4 and 3 when only the angles inside the Snell cone are considered. This is the range of values expected for the water-leaving radiances.

The images in Fig. 7 are individual snapshots, which sometimes include artifacts. The instrument cable appears, for instance, at the horizon for example II $(\lambda=$ $628 \mathrm{~nm} ; 135^{\circ}$ azimuth). It was mentioned above that the shadows of the floatation arms sometimes appear. Enhanced values near the horizontal direction are sometimes due to the lens effects of surface waves. These surface effects and the occasional presence of the cable in the field of view can easily be filtered out or averaged by combining several successive images.

\section{b. $4 \pi$ radiance distributions at depth}

A key feature of the CE600 camera system is its ability to measure radiances over a large range of intensities. This capability allows simultaneous measurements of $L\left(\Xi_{u}\right)$ and $L\left(\Xi_{d}\right)$ at varying depths in the water column. To explore this possibility of getting in-water vertical profiles of the radiance distribution over $4 \pi \mathrm{sr}$, a prototype profiling system was built. The design is similar to that of commercially available profiling radiometers, with two cameras head-to-tail formation at each end of a rocket-shape body whose upper section is equipped with four stabilizing fins. This profiling system is deployed in a free-fall mode at a distance from the ship that ensures the ship hull is not seen (or represents a negligible perturbation). Acquisition sequences [both $L\left(\Xi_{u}\right)$ and $\left.L\left(\Xi_{d}\right)\right]$ are sequentially performed during the descent.

An example is provided in Fig. 9, using data collected under a clear sky on 23 August 2009 in clear waters of the Arctic $\left(\lambda=494 \mathrm{~nm}, \theta_{s}=65^{\circ}\right.$; see figure caption for details). The $L\left(\Xi_{d}\right)$ close to the surface (upper left plot) is irregularly shaped because of the surface wave effects (no swell; only small capillary waves). The imprint of waves progressively disappears with increasing depths and the shape of the radiance contours moves from ellipses to circles as the rearrangement of the light field occurs. The maximum range of downwelling radiances is 
Clear water, clear sky, $\theta_{\mathrm{s}} 27^{\circ} 4$ Clear water, clear sky, $\theta_{\mathrm{s}} 60^{\circ} 5 \quad$ Turbid, clear sky, $\theta_{\mathrm{s}} 38^{\circ} 4$

Turbid, overcast, $\theta_{\mathrm{s}} 54^{\circ} 6$
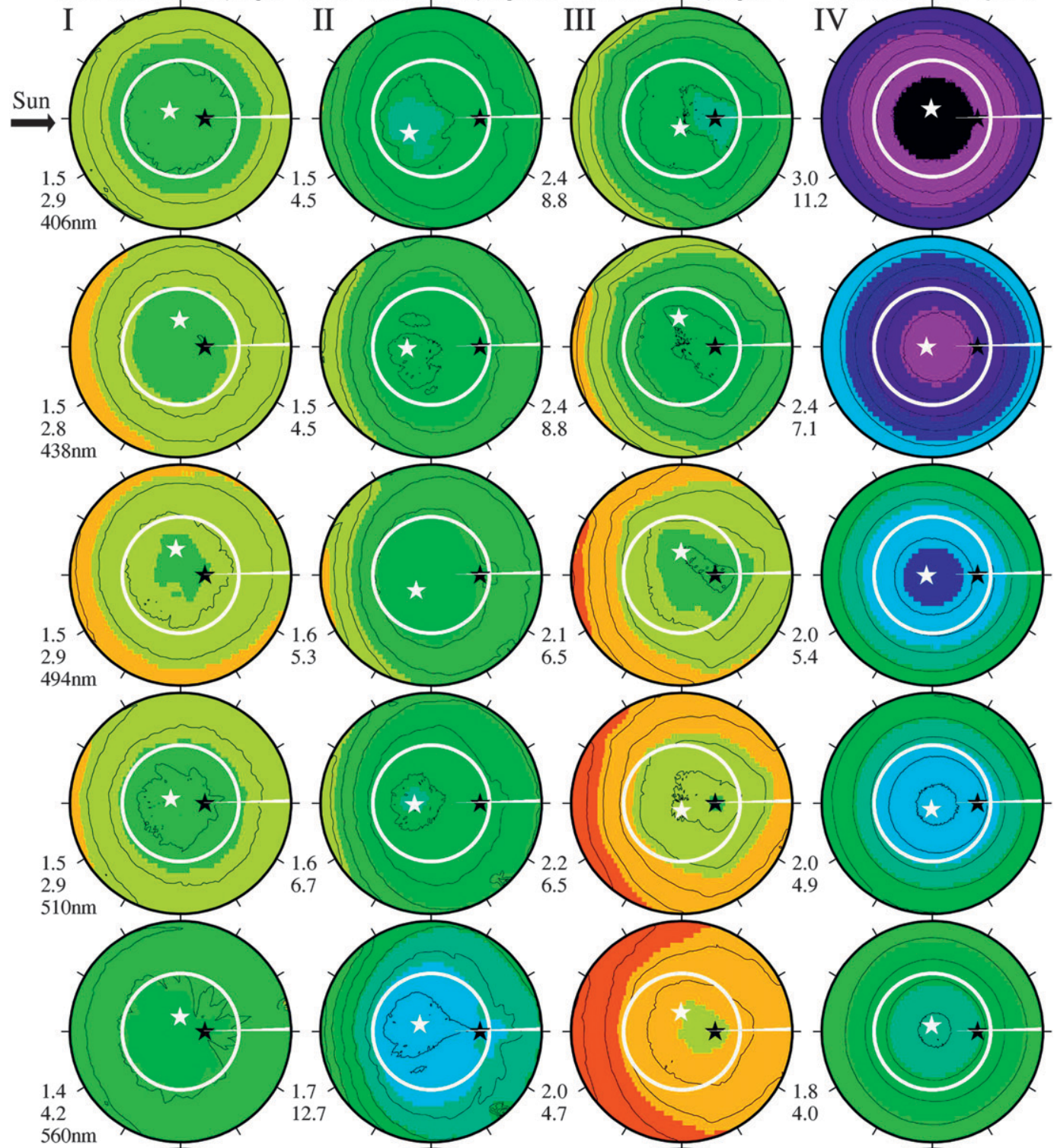

$560 \mathrm{~nm}$
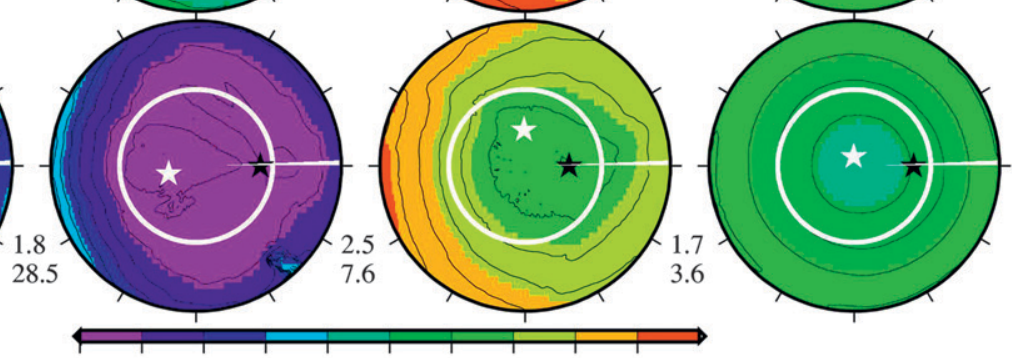

$\begin{array}{llll}0.01 & 0.03 & 0.06 & 0.1\end{array}$

$\mathrm{L}_{\mathrm{u}}\left(\mu \mathrm{W} \mathrm{cm} \mathrm{mm}^{-1} \mathrm{sr}^{-1}\right)$

FIG. 7. Sample $L\left(\Xi_{u}, 0^{-}\right)$distributions, for the six camera spectral bands and four environment conditions, as indicated on top of each column. The roman numerals on top refer to the descriptions given in Table 6 . The center of these polar plots is the nadir direction, and their circumference is the horizon. Relative azimuth angles with respect to the sun azimuth go counterclockwise from zero (sun side) on the left side of the horizontal diameter. The principal plane is the horizontal diameter. The black star is the antisolar point and the white star is where the minimum radiance was found in the image. The inner white circle delineates the critical angle. The two numbers on the lower left corner of each plot are the range of radiance values found in the entire image (first value above the wavelength) and within the Snell cone (corresponding to those radiances that exit the ocean). 
TABLE 6. Environmental conditions and optical properties for the four examples shown in Fig. 7 (roman numerals given here correspond to those on top of each column of Fig. 7).

\begin{tabular}{|c|c|c|c|c|c|c|c|c|}
\hline & Selected case & Location & Acquisition time & Water type & Sky & $\theta_{s}\left({ }^{\circ}\right)$ & $\operatorname{Chl}\left(\mathrm{mg} \mathrm{m}^{-3}\right)$ & $\mathrm{b} / \mathrm{c}^{*}(412-670 \mathrm{~nm})$ \\
\hline I & Boussole & NW Mediterranean Sea & $\begin{array}{l}1127 \text { UTC } \\
18 \text { Jul } 2008\end{array}$ & Clear & Clear & 27.4 & 0.3 & $\sim 0.82-0.23$ \\
\hline II & $\begin{array}{l}\text { Malina } \\
\quad \text { station } 235\end{array}$ & Beaufort Sea, Arctic & $\begin{array}{l}2017 \text { UTC } \\
23 \text { Aug } 2009\end{array}$ & Clear & Clear & 60.5 & 0.1 & $\sim 0.79-0.12$ \\
\hline III & $\begin{array}{l}\text { OpticMed } \\
\text { station } 8\end{array}$ & $\begin{array}{l}\text { NW Mediterranean Sea, } \\
\text { Rhone River plume }\end{array}$ & $\begin{array}{l}0928 \text { UTC } \\
5 \text { May } 2008\end{array}$ & $\begin{array}{l}\text { Moderately } \\
\text { turbid }\end{array}$ & Clear & 38.4 & 0.3 & $\mathrm{~N} / \mathrm{A} * *$ \\
\hline IV & $\begin{array}{l}\text { Malina } \\
\text { station } 696\end{array}$ & Beaufort Sea, Arctic & $\begin{array}{l}2134 \text { UTC } \\
13 \text { Aug } 2009\end{array}$ & Highly turbid & Overcast & 54.6 & 3 & N/A \\
\hline
\end{tabular}

* The particulate scattering coefficient $b_{p}$ is here computed from Chl following Loisel and Morel (1998). The seawater scattering coefficient $b_{w}$ is computed from Morel (1974). These two coefficients are summed up to give the total scattering coefficient $b$. The particulate absorption $a_{p}$ is determined from Chl following Bricaud et al. (1998). Colored dissolved organic matter (CDOM) absorption $a_{\text {cdom }}$ is determined from Chl following Morel and Gentili (2009). Pure water absorption $a_{w}$ is taken from Pope and Fry (1997) and Morel et al. (2007). These three partial coefficients are summed up to get total absorption.

** Cannot be computed here because data are missing.

found here for $z=36 \mathrm{~m}$ (the maximum to minimum ratio is $\sim 1700$ ) and not, as intuitively expected, just beneath the surface. Close to beneath a flat sea surface, low (minimum) radiances would occur for directions near the horizon, creating a very large contrast with the maximum radiance found in the sun's direction. At these shallow depths, radiances near the horizontal are, however, reinforced by the refraction of direct sunlight by capillary waves, decreasing the range of radiances as compared to greater depths where this phenomenon does not occur. The refracted image of the sun still clearly shows up at the deepest measurement depth (90 m), which is slightly below the $1 \%$ light level at this wavelength. Downwelling radiances at this depth still span three orders of magnitude from the horizontal to the direction of the refracted direct sunrays, which is more than what was expected and shows that the optical depth is still not large enough for the asymptotic regime to be approached (e.g., Morel and Gentili 2004). In contrast, the $L\left(\Xi_{u}\right)$ distributions are smooth. The upwelling flux at the greatest depth $(z=90 \mathrm{~m})$ is nearly isotropic. The dim radiances at this depth were well captured by the instrument, using a 500-ms integration time.

\section{Validation through comparisons with other instruments}

The acquisition of in-water high-accuracy radiometric measurements from fish-eye cameras has rarely been attempted and includes significant challenges. That is the reason for the care taken in characterizing and calibrating our cameras (results in previous sections). These challenges call for a qualification of our measurements in comparison to those of well-known and proven instruments, which is the aim of the experiments reported below.
Comparisons have been carried out between nadir upwelling radiances measured by the camera just beneath the surface, $L_{u}\left(\theta=\pi, 0^{-}\right)$, and their values extrapolated from in-water profiles of the nadir upwelling radiance, $L_{u}(\theta=\pi, z)$. These comparisons were performed using data from two commercial in-water profiling
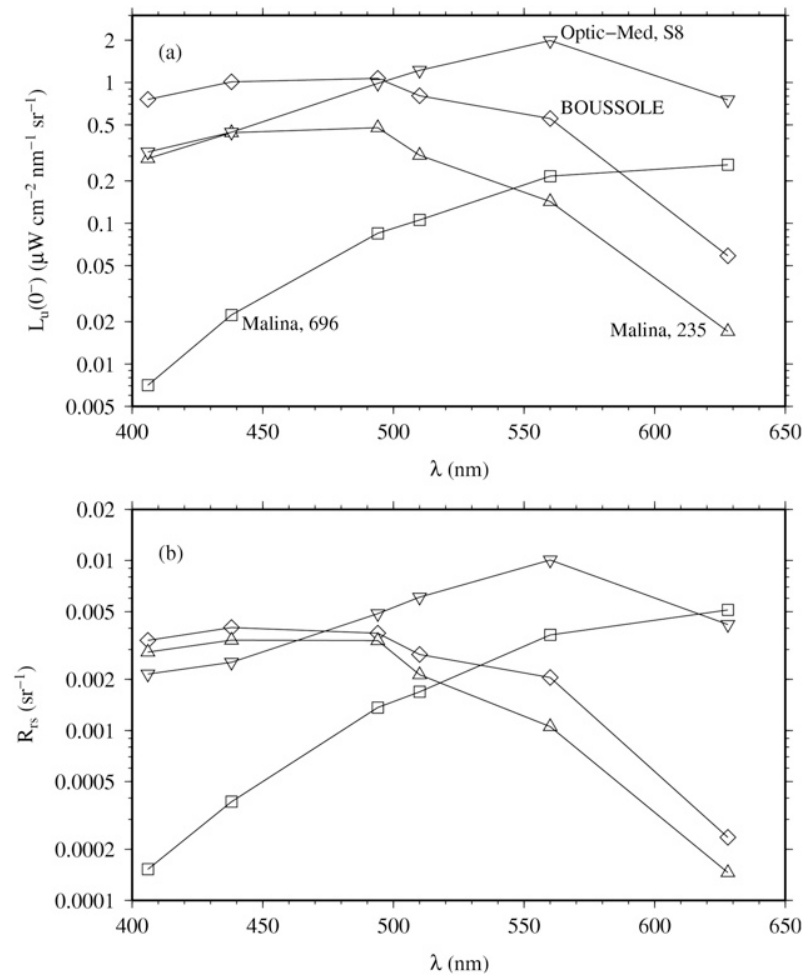

FIG. 8. Spectra of the (a) upwelling nadir radiance just below the surface $L_{u}\left(0^{-}\right)$and (b) remote sensing reflectance RSR for the four selected situations used in Fig. 7, as indicated. The RSR is computed as $L_{w} / E_{s}$, where $E_{s}$ was measured parallel to the camera measurements by a Biospherical C-OPS deck reference (Morrow et al. 2010). 
$\mathrm{L}\left(\Xi_{\mathrm{d}}\right)$

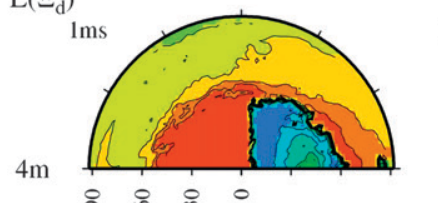
\& 8 \& 0
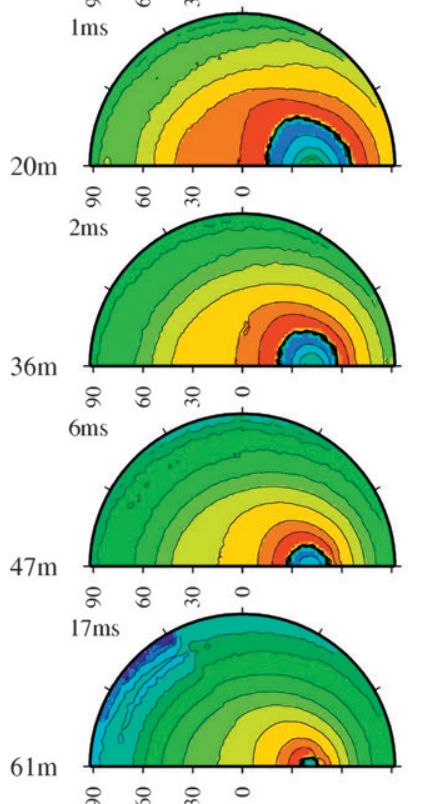

\& 8 \& 0

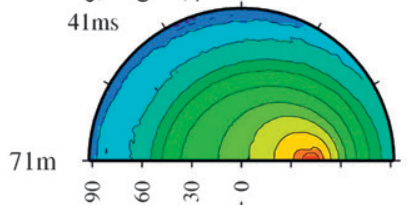

\& \&

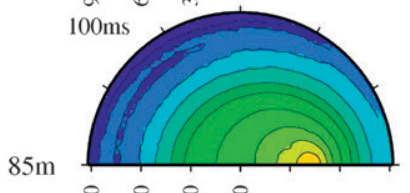

\& \&
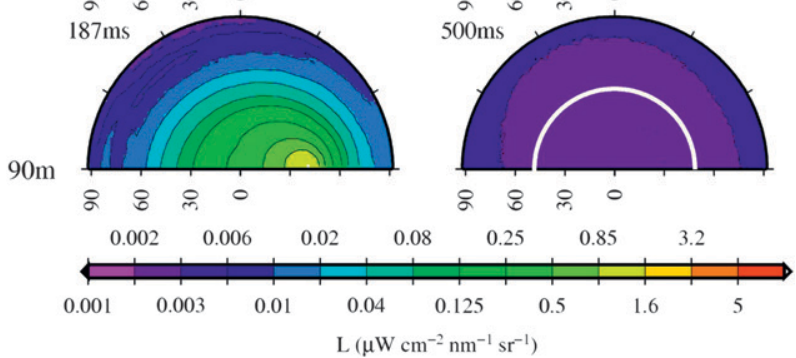

FIG. 9. The (left) $L\left(\Xi_{d}, z\right)$ and (right) $L\left(\Xi_{u}, z\right)$ for the depths indicated, for $\lambda=494 \mathrm{~nm}$, and for integration times as indicated (ms). Data were collected in the Arctic (Beaufort Sea) during the Malina cruise in August 2009 (station 235-2). The sun zenith angle was $65^{\circ}$, the sky was clear, and the chlorophyll concentration was $0.1 \mathrm{mg}(\mathrm{Chl}) \mathrm{m}^{-3}$. For the $L\left(\Xi_{u}, z\right)$ distributions, the inner white circle delineates the critical angle. For the five $L\left(\Xi_{d}, z\right)$ distributions corresponding to depths from 4 to $61 \mathrm{~m}$, the highest values around the sun image have been divided by 1000 so as not to have to use different color coding. They appear in blue and green colors. radiometers. The first one is a Satlantic microPRO profiling radiometer system (Zibordi et al. 2004, 2011) deployed during a cruise in the Mediterranean Sea in October 2008 (LSCV08 cruise). The second one is a Biospherical Compact Optical Profiling System (C-OPS; Morrow et al. 2010) deployed in August 2009 in the Beaufort Sea in the Arctic [Mackenzie light and carbon (MALINA) cruise]. Deployment procedures and extrapolation techniques follow the recommended ocean optics protocols (Mueller et al. 2003).

The correlation between the camera-derived $L_{u}(\theta=$ $\left.\pi, 0^{-}\right)$and those derived from the microPRO $L_{u}(\theta=\pi, z)$ profiles (Fig. 10a) is excellent ( $r^{2}$ close to 1$)$, with very little dispersion (RMSE $=0.09$; both instruments were deployed simultaneously). The correlation with the C-OPS-derived $L_{u}\left(\theta=\pi, 0^{-}\right)$is slightly lower (Fig. 10b), yet still very good $\left(r^{2}=0.965\right)$. In this case the camera was not deployed simultaneously with the in-water profiler. The small time lag (usually $\sim 15 \mathrm{~min}$ ), although accounted for by correcting the data for the change of the cosine of the sun zenith angle, likely explains the higher dispersion. One of the C-OPS casts was used to compare the nadir upwelling radiance at various depths from just beneath the surface down to $90 \mathrm{~m}$ (Fig. 10c; same profile as in Fig. 9). The agreement is also excellent $\left(r^{2}=0.98\right)$ over nearly four orders of magnitude in radiance.

Finally, the upwelling radiance distribution measured just beneath the sea surface by the CE600 has been compared to the same distribution measured by the NuRADS camera (Voss and Chapin 2005). Here the radiances were normalized to their value at nadir (Fig. 10d). The agreement between the two cameras is very good $\left(r^{2}=0.98\right)$. Overall, the agreement obtained here when comparing the CE600 measurements to those of other radiometers shows the excellent radiometric quality of the CE600.

\section{Apparent and inherent optical properties derived from vertical profiles of $L\left(\Xi_{\mathrm{u}}\right)$ and $L\left(\Xi_{\mathrm{d}}\right)$}

The potential of using vertical profiles of $L\left(\Xi_{u}\right)$ and $L\left(\Xi_{d}\right)$ for the derivation of integrated radiometric quantities (e.g., irradiances) and of AOPs and IOPs has been explored using the data shown in Fig. 9 (Malina station 235).

At a given wavelength, the scalar irradiance $\stackrel{o}{E}$ is obtained by integrating the radiance distribution ( $\lambda$ omitted) over the whole space, so that

$$
\stackrel{o}{E}=\int_{\Xi} L(\theta, \phi) d \Omega .
$$

If the integration is performed over the half-space corresponding to the upper hemisphere, that is, over 

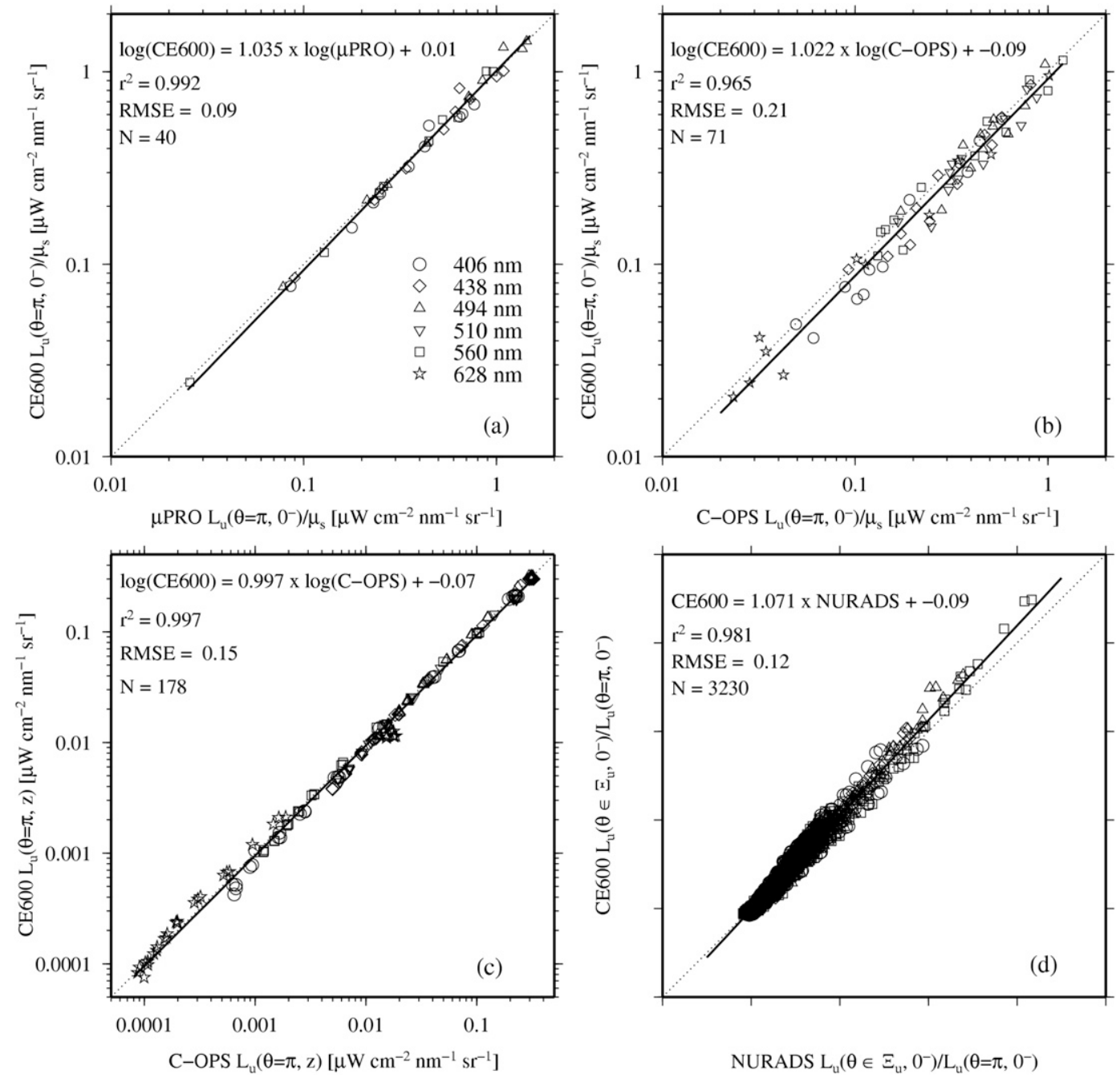

FIG. 10. (a) The $L_{u}\left(\theta=\pi, 0^{-}\right)$(i.e., nadir $\left.L_{u}\right)$ from the CE600 camera vs $L_{u}\left(\theta=\pi, 0^{-}\right)$from a MicroPRO radiometer, for the spectral bands indicated. The data were collected in the Mediterranean (Ligurian Sea) during the LSCV08 cruise in October 2008 (stations 16-22, 25, 27, and 28). The dotted line is the 1:1 line. The solid line is a linear fit on the log-transformed data, whose parameters are given on top. (b) As in (a), but using data from a Biospherical C-OPS profiling radiometer system. Data were collected in the Arctic (Beaufort Sea) during the Malina cruise in August 2009 (stations 150, 170, 240, 260, 280, 320, 360, 394, 395, 396, 398, and 691). (c) As in (b), but for $L_{u}(\theta=\pi, z$ ) from $z=0^{-}$(just beneath the surface) down to $z=90 \mathrm{~m}$ (Malina station 235-1). (d) The $L_{u}\left(\theta \in \Xi_{u}, 0^{-}\right) / L_{u}\left(\theta=\pi, 0^{-}\right.$) from the CE600 camera vs $L_{u}\left(\theta \in \Xi_{u}, 0^{-}\right) / L_{u}\left(\theta=\pi, 0^{-}\right)$from NuRADS (Voss and Chapin 2005). The data were collected in the Mediterranean during the LSCV08 cruise in October 2008.

$\Xi_{d}$ (corresponding to downwelling photons), or over the lower hemisphere, $\Xi_{u}$ (upwelling photons), the downward scalar irradiance or upward scalar irradiance is obtained and denoted

$$
\stackrel{o}{E_{d}} \text { and } \stackrel{o}{E}_{u} \text {,respectively. }
$$

The downward irradiance on a horizontal plane $E_{d}$ is the integral of all downwelling radiances intercepting an element of this plane surface, so that

$$
E_{d}=\int_{\Xi d} L(\theta, \phi) \cos \theta d \Omega
$$

Similarly, the upward planar irradiance $E_{u}$ is obtained as

$$
E_{u}=\int_{\Xi u} L(\theta, \phi)|\cos \theta| d \Omega,
$$

where the absolute value $|\cos \theta|$ is used to obtain a positive quantity for $E_{u}$. The difference 


$$
E_{d}-E_{u}=\int_{\Xi} L(\theta, \phi) \cos \theta d \Omega
$$

represents the "net downward flux," and is the modulus of the vector irradiance $\mathbf{E}$ vertically oriented downward. The ratio $E_{u} / E_{d}$, where $E_{u}$ and $E_{d}$ are defined (measured) at the same depth, is the irradiance reflectance $R$ at this depth.

From the above planar and scalar irradiances, it is possible to derive the average cosines for downward and upward irradiances, respectively (their inverse being referred to as the distribution functions):

$$
\bar{\mu}_{d}=\frac{E_{d}}{\stackrel{o}{E}_{d}} \text { and } \bar{\mu}_{u}=\frac{E_{u}}{\stackrel{E}{o}_{u}} .
$$

Before integrating $L\left(\Xi_{u}\right)$ and $L\left(\Xi_{d}\right)$ to get the scalar, planar, and net irradiances [Eqs. (3)-(7)], missing data in the $L\left(\Xi_{d}\right)$ distributions close to the image of the sun had to be reconstructed. These missing data correspond to the few saturated pixels in the direction of the direct sunrays and for small-angle scattering around this direction. Letting these pixels saturate (i.e., not decreasing the integration time too much) allowed the rest of the $L\left(\Xi_{d}\right)$ distribution to be accurately measured. Close to the surface, these missing data can significantly contribute to the downward irradiance (up to $5 \%$ for the cast used here), hence the need for filling them. The reconstruction used a two-dimensional Gaussian fit to the logarithm of the data surrounding the area with missing data. This fit is constrained so the reconstructed distribution includes the approximate value of the radiance of the direct underwater sunbeam at depth $z$, $L_{d}\left(\lambda, z, \theta^{\prime}\right)$, where $\theta^{\prime}$ is the refracted sun zenith angle $\left(\theta_{s}\right)$. This radiance is determined as

$$
L_{d}\left(\lambda, z, \theta^{\prime}\right)=\frac{F_{0}(\lambda)}{\Omega} t_{r}\left(\lambda, \theta_{S}\right) n^{2} e^{-K_{L}\left(\lambda, \theta^{\prime}\right) z},
$$

where $F_{0}(\lambda)$ is the extraterrestrial irradiance (Thuillier et al. 1998), $\Omega$ is the apparent solid angle of the sun $(7 \times$ $10^{-5} \mathrm{sr}$ ) (so $F_{0} / \Omega$ is the radiance of the direct sun in direction $\left.\theta_{s}\right), t_{r}\left(\lambda, \theta_{s}\right)$ is the transmittance of the atmosphere due to molecular scattering only (computed as $e^{\left[-\tau_{r} / 2 / \cos \left(\theta_{s}\right)\right]}$, where $\tau_{r}$ is the Rayleigh scattering optical thickness), $n^{2}$ is the square of the water refractive index (transmission across the interface), and $K_{L}\left(\lambda, \theta^{\prime}\right)$ is the diffuse attenuation coefficient for the downwelling radiance around the sun's direction (determined from the data themselves). The last processing step consisted of connecting the $L\left(\Xi_{u}\right)$ and $L\left(\Xi_{d}\right)$ distributions by adjusting $L\left(\Xi_{d}\right)$ so as to get the same average radiance over the horizon (i.e., $\theta>89^{\circ}$ and $\phi \in 0^{\circ}-360^{\circ}$ ) as in $L\left(\Xi_{u}\right)$.

oThe various irradiances $\left[E_{u}(z), \stackrel{o}{E_{u}}(z), E_{d}(z)\right.$, and $\left.E_{d}(z)\right]$ were subsequently interpolated onto a 1 -m-depth grid (log interpolation). The resulting profiles are shown in Fig. 11a, along with the nadir $Q$ factor [i.e., the ratio $\left.E_{u} / L_{u}(\theta=\pi)\right]$. The profiles do not start just beneath the sea surface because the first $L\left(\Xi_{d}\right)$ measurement was taken at $13 \mathrm{~m}$. They show the expected logarithmic decrease with depth. Because the data were collected at depths spaced largely apart (black stars in Fig. 11a), these interpolated profiles cannot show changes in attenuation that would be due to local changes in optical properties. A denser acquisition would be necessary to get a finer description of these AOPs along the vertical. The main interest in simultaneously getting $E_{u}(z)$, $\stackrel{o}{E}_{u}(z), E_{d}(z)$, and ${ }_{E_{d}}(z)$ is that all AOPs can be determined, including diffuse attenuation coefficients for irradiances or radiances and reflectance (not shown here). The "average cosines" [Eq. (8)] are also derivable. They are displayed in Fig. 11b. Their values are as expected, with $\bar{\mu}_{d}$ in the $0.7-0.8$ range and increasing with depth (open circles), and $\bar{\mu}_{u}$ slightly larger than 0.4 and also increasing with depth (open triangles).

A further step is to combine these AOPs to retrieve IOPs, namely, the total absorption coefficient $a$ and the backscattering coefficient $b_{b}$. By doing so, the measurement scale of both IOPs and AOPs is reconciled, which is not the situation when using conventional IOP instrumentation that samples at a scale of a few centimeters cubed only when the radiometric measurements inherently integrate a much larger volume (several meters cubed). This is one advantage of AOP inversion (Gordon 2002). Besides this scale mismatch, a weakness of currently used IOP instrumentation is that it does not refer to any measurement standard. Deriving IOPs from AOPs would therefore tie all optical properties to the same radiometric scale.

The derivation of absorption was performed through the law of conservation of energy, expressed as Gershun's law (Gershun 1939). The following form of this expression was used here (see also Voss 1989b):

$$
a(z) \stackrel{o}{E}(z)=-\frac{d\left[E_{d}(z)-E_{u}(z)\right]}{d z} .
$$

This equation is valid without internal radiative sources such as Raman scattering or fluorescence (in other words without inelastic scattering). The resulting profile of the absorption coefficient is displayed in Fig. 11b (gray circles), showing rather constant values at depth. As said above for the irradiance profiles, the sparse sampling at depth for this specific cast cannot provide information on 

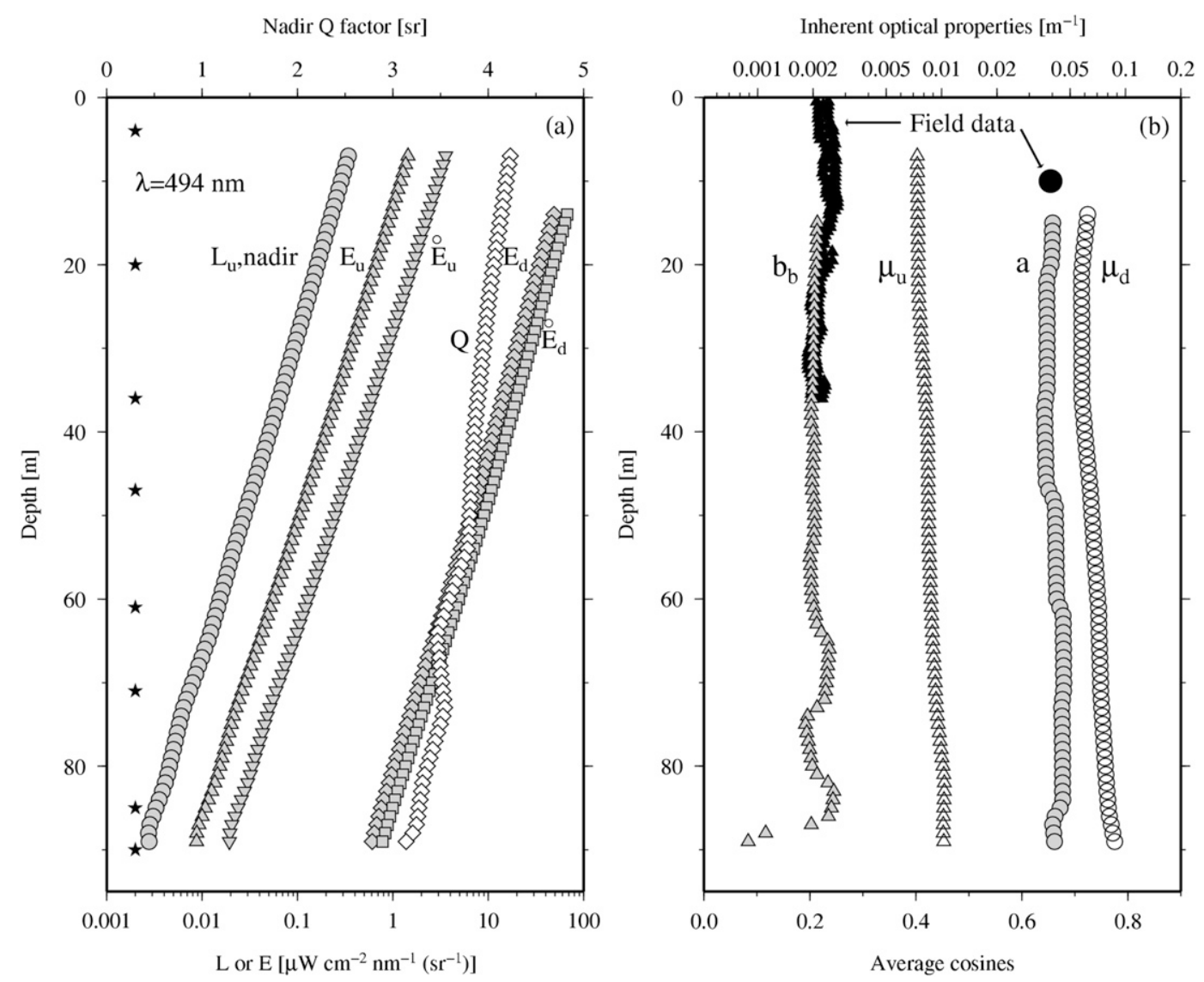

FIG. 11. (a) Vertical profiles of the nadir upwelling radiance $L_{u}(\theta=\pi, z)$, and of $E_{u}(z), \stackrel{o}{E_{u}}(z), E_{d}(z), \stackrel{o}{E_{d}}(z)$, and $Q$ (see text). (b) Vertical profiles of $\bar{\mu}_{d}$ and $\bar{\mu}_{u}$ (open triangles and circles; scale on the lower axis), and of $b_{b}$ and $a$ (gray triangles and circles; scale on the upper axis), as derived from the profiles of radiometric quantities shown in (a) (see text). The black symbols are for the backscattering and absorption coefficients as determined in situ from conventional IOP instrumentation.

possible small-scale structures. The small step changes that are visible correspond to inaccuracies introduced by the interpolation to a regular 1-m grid. These absorption values obtained by inversion are compared to an independently determined absorption coefficient (black circle). This one was obtained by summing up pure seawater absorption (Morel et al. 2007), particulate absorption determined in situ from filtered samples (Bricaud et al. 1995), and absorption by colored dissolved organic matter determined using a multiple pathlength spectrophotometer (Miller et al. 2002; Bricaud et al. 2010). The water for these analyses was taken at a depth of $10 \mathrm{~m}$. This single comparison shows excellent agreement between the conventionally measured absorption coefficient and its value obtained from the inversion of AOPs.

The backscattering coefficient was also determined, using an inversion scheme previously proposed by Zaneveld (1989). This method is based on the asymptotic closure theory, which uses some specific properties of the radiant field at large optical depths. It is out of scope here to fully describe the rationale for this theory and the mathematical derivation that leads to the equation used here [Eq. (37) of Zaneveld (1989)]:

$$
b_{b}(z)=\frac{\operatorname{RSR}(z)[K(\pi, z)+a(z)]}{(1 / 2 \pi)-\operatorname{RSR}(z)}
$$

where [Eqs. (35) and (38) in Zaneveld (1989)]

$$
\operatorname{RSR}(z)=\frac{L(\pi, z)}{E_{d}(z)} \text { and } K(\pi, z)=-\frac{1}{L(\pi, z)} \frac{d L(\pi, z)}{d z}
$$

where $K(\pi, z)$ is the diffuse attenuation coefficient for the nadir upwelling radiance at depth $z$, and $\operatorname{RSR}(z)$ is referred to in Zaneveld (1989) as the remotely sensed 
TABLE 7. Absorption and backscattering coefficients $\left(\mathrm{m}^{-1}\right)$, derived from direct measurements or from AOP inversion (see section 7).

\begin{tabular}{|c|c|c|c|c|c|}
\hline & & \multicolumn{2}{|c|}{ Absorption coefficient } & \multicolumn{2}{|c|}{ Backscattering coefficient } \\
\hline & & Direct measurements & AOP inversion & Direct measurements & AOP inversion \\
\hline \multirow[t]{2}{*}{$406 \mathrm{~nm}$} & Total & 0.0729 & 0.0805 & $4.21 \times 10^{-3}$ & $3.70 \times 10^{-3}$ \\
\hline & Total minus water & 0.0683 & 0.0759 & $1.11 \times 10^{-3}$ & $6.02 \times 10^{-4}$ \\
\hline \multirow[t]{2}{*}{$438 \mathrm{~nm}$} & Total & 0.0505 & 0.0469 & $2.91 \times 10^{-3}$ & $2.99 \times 10^{-3}$ \\
\hline & Total minus water & 0.0445 & 0.0410 & $6.73 \times 10^{-4}$ & $7.54 \times 10^{-4}$ \\
\hline \multirow[t]{2}{*}{$494 \mathrm{~nm}$} & Total & 0.0391 & 0.0384 & $2.26 \times 10^{-3}$ & $2.06 \times 10^{-3}$ \\
\hline & Total minus water & 0.0218 & 0.0211 & $9.19 \times 10^{-4}$ & $7.11 \times 10^{-4}$ \\
\hline \multirow[t]{2}{*}{$560 \mathrm{~nm}$} & Total & 0.0711 & 0.0675 & $1.41 \times 10^{-3}$ & $1.50 \times 10^{-3}$ \\
\hline & Total minus water & 0.0092 & 0.0056 & $6.14 \times 10^{-4}$ & $7.10 \times 10^{-4}$ \\
\hline
\end{tabular}

reflectance at depth $z$ (the terminology "remote sensing reflectance" is used when RSR is derived from quantities just above the sea surface, i.e., the water-leaving radiance and the downward solar irradiance).

All quantities in Eqs. (11) and (12) can be derived from the CE600 camera measurements. The resulting vertical profile of the backscattering coefficient is displayed in Fig. 11b (gray triangles). The black triangles in Fig. 11b are two independent measurements of the same quantity, using commercial backscattering meters (a Hobilabs Hydroscat-6 and a Wetlabs EcoBB3). The agreement between the AOP-derived and the conventionally measured backscattering coefficients is excellent, especially considering that this test was performed for clear waters with a low particle load. Average values in the top $30 \mathrm{~m}$ are given in Table 7 for four of the camera spectral bands [the vertical resolution of the $L(\Xi)$ profile did not allow inversion to be performed at $628 \mathrm{~nm}$. The agreement is within $10 \%$ for total absorption and within $12 \%$ for total backscattering, which is excellent considering the poor resolution of the $L(\Xi)$ profiles. More comprehensive testing of the inversion scheme still has to be performed, in particular for different particle loads and by increasing the vertical resolution of $L(\Xi)$ measurements.

\section{Conclusions}

A new instrument for the measurement of underwater radiance distributions has been developed, characterized, calibrated, and deployed in various environments. The initial results presented in this paper show the high radiometric quality of this radiance camera, and its potential in providing measurements of the underwater radiance distribution over a hemisphere in six spectral bands. When two of these cameras are used simultaneously, the underwater radiance distribution is obtained over the entire space (i.e., $4 \pi$ sr). This capability allows all optical properties to be determined from a single and well-calibrated couple of instruments.
The data shown here are among the first of that sort ever obtained [but see the concurrent development of Lewis et al. (2011)]. Numerous research domains will benefit from such measurements, including optical closure [deriving IOPs and AOPs from a unique set of $L(\Xi, z)]$ and characterization of the bidirectionality of the ocean reflectance in Case-2 waters (where currently there are virtually no data). Other potential applications would benefit from the $L\left(\Xi_{d}\right)$ measurements for smallangle scattering away from the direct sun to analyze the forward part of the volume scattering function and its relation to the particle size distribution. The characteristics of the air-sea interface can also be explored using $L(\Xi)$ changes with depth and time close to the surface (You et al. 2011).

For these developments to become reality, further characterization, calibration, and validation are still needed and are actually ongoing. These include the inwater determination of the geometrical projection and rolloff function, evaluation of stray-light contamination, and determination of the point-spread function (PSF). A final error budget will be produced after all aspects of the characterization and calibration are fully understood and quantified. The excellent validation results shown here when comparing to well-calibrated commercial radiometers already demonstrate that high radiometric accuracy is reached.

Ongoing or future improvements in terms of the instrument design or deployment protocols include the addition of a deck reference (at least a planar irradiance sensor for the correction of underwater measurements for changes in above-surface irradiance) and the development of a profiling system including buoyancy trimming, which is needed to accurately adjust the descent speed. Another major adaptation consists of using the camera for sky radiance measurements (see, e.g., Zibordi and Voss 1989). Such measurements are mostly performed using scanning sun and sky photometers, such as the CIMEL CE318 that equips the AERONET world aerosol measurement network (Holben et al. 
1998). These instruments have many advantages for such a purpose, yet they have the major disadvantage of requiring significant time to perform measurements in only the principal plane and one almucantar. Sky conditions may change during this time (10-15 min), preventing an accurate interpretation of the measurements. A radiance camera aimed at the sky totally suppresses this inconvenience by providing an instantaneous measure of radiances in all directions. Adaptations of the present instrument are, however, necessary, essentially to cope with the presence in the image field of the bright sun image. This image actually has to be blocked to avoid saturation and to maintain the significantly long integration time needed to get an accurate measure of the lower sky radiances. The temperature regulation has to be improved as well, by using a dissipating radiator. Initial tests in such a configuration have shown excellent comparisons with a scanning photometer simultaneously operated, indicating a low level of stray light in the camera.

Acknowledgments. This paper is dedicated to the memory of one of our co-authors, Professor André Morel, who passed away on 23 September 2012. He was a pioneer of ocean optics and ocean color science, and a mentor for many of us in the ocean optics community. His work on the directionality of the ocean reflectance has largely inspired the instrumental development that is presented in this paper.

We thank the two anonymous reviewers for their comments on the initial manuscript. Stanford Hooker, Kenneth Voss, and Giuseppe Zibordi are warmly thanked for having graciously provided their data for comparison with the CE600 measurements (respectively, the Biospherical C-OPS, the NuRADS radiance camera, and the Satlantic microPRO data). The joint development of the CE600 radiance camera by CIMEL Electronique and the Marine Optics group of the Laboratoire d'Océanographie de Villefranche (LOV) was initiated in 2004 thanks to a "Research and Technology" (R\&T) grant from the Centre National d'Etudes Spatiales (CNES; French Space Agency). Continuing support of CNES since 2004 is acknowledged. Funding was also obtained from the Institut National des Sciences de l'Univers (INSU) for development of a radiometric calibration facility.

\section{REFERENCES}

Aas, E., and N. K. Højerslev, 1999: Analysis of underwater radiance observations: Apparent optical properties and analytic functions describing the angular radiance distribution. J. Geophys. Res., 104, 8015-8024.

Adams, J. T., E. Aas, N. K. Hjøerslev, and B. Lundgren, 2002: Comparison of radiance and polarization values observed in the Mediterranean Sea and simulated in a Monte Carlo model. Appl. Opt., 41, 2724-2733, doi:10.1364/AO.41.002724.

Bhandari, P., K. J. Voss, and L. Logan, 2011a: An instrument to measure the downwelling polarized radiance distribution in the ocean. Opt. Express, 19, 17 609-17 620.

$-, \ldots, \ldots$, and M. Twardowski, 2011b: The variation of the polarized downwelling radiance distribution with depth in the coastal and clear ocean. J. Geophys. Res., 116, C00H10, doi:10.1029/2011JC007320.

Bricaud, A., M. Babin, A. Morel, and H. Claustre, 1995: Variability in the chlorophyll-specific absorption coefficient of natural phytoplankton: Analysis and parameterization. J. Geophys. Res., 100, 13 321-13 332.

— A. Morel, M. Babin, K. Allali, and H. Claustre, 1998: Variations of light absorption by suspended particles with the chlorophyll a concentration in oceanic (Case 1) waters: Analysis and implications for bio-optical models. J. Geophys. Res., 103, 31 033-31 044.

—, M. Babin, H. Claustre, J. Ras, and F. Tieche, 2010: Light absorption properties and absorption budget of South East Pacific waters. J. Geophys. Res., 115, C08009, doi:10.1029/ 2009JC005517.

Gershun, A. A., 1939: The light field. J. Math. Phys. (Cambridge, Mass.), 18, 51-151.

Gleason, A. C. R., and Coauthors, 2012: Detailed validation of the bidirectional effect in various Case I and Case II waters. Opt. Express, 20, 7630-7645.

Gordon, H. R., 2002: Inverse methods in hydrologic optics. Oceanologia, 44, 9-58.

- and W. R. McCluney, 1975: Estimation of the depth of sunlight penetration in the sea for remote sensing. Appl. Opt., 14, 413-416.

, O. B. Brown, and M. J. Jacobs, 1975: Computed relationships between the inherent and apparent optical properties of a flat homogeneous ocean. Appl. Opt., 14, 417-427.

Haltrin, V. I., M. E. Lee, and O. V. Martynov, 1997: Rapid hydrologic measurements of underwater angular distribution of light. Proc. 12th Int. Conf. on Applied Geologic Remote Sensing, Vol. 1, Denver, CO, Environmental Research Institute of Michigan, 361-368.

Hojerslev, N. K., 1989: A history of early optical oceanographic instrument design in Scandinavia. Ocean Optics, R. W. Spinrad, K. L. Carder, and M. J. Perry, Eds., Oxford University Press, $118-147$.

Holben, B. N., and Coauthors, 1998: AERONET - A federated instrument network and data archive for aerosol characterization. Remote Sens. Environ., 66, 1-16.

Ivanoff, A., and J.-L. Moreuil, 1963: Au sujet de la répartition des luminances sous-marines au voisinage immédiat de la direction apparente du soleil. C. Roy. Acad. Sci., Paris, 256, 4711-4712.

Jerlov, N. G., 1976: Marine Optics. Elsevier Scientific, 231 pp.

- , and G. Liljequist, 1938: On the Angular Distribution of Submarine Daylight and on the Total Submarine Illumination. Svenska Hydrografisk-Biologiska Kommissionens Skrifter: Hydrografi Series, Vol. 14, Hakan Ohlsson, 15 pp.

—, and M. Fukuda, 1960: Radiance distribution in the upper layers of the sea. Tellus, 12, 348-355, doi:10.1111/j.21533490.1960.tb01319.x.

Johnson, B. C., S. S. Bruce, E. A. Early, J. M. Houston, T. R. O'Brian, A. Thompson, S. B. Hooker, and J. L. Mueller, 1996: The Fourth SeaWiFS Intercalibration Round-Robin Experiment (SIRREX-4), May 1995. Tech. Memo. 104566, Vol. 37, NASA GSFC, Greenbelt, MD, 65 pp. 
Kirk, J. T. O., 1981: Monte Carlo study of the nature of the underwater light field in, and the relationships between optical properties of, turbid yellow waters. Aust. J. Mar. Freshwater Res., 32, 517-532.

Lewis, M. R., J. Wei, R. Van Dommelen, and K. J. Voss, 2011: Quantitative estimation of the underwater radiance distribution. J. Geophys. Res., 116, C00H06, doi:10.1029/2011JC007275.

Loisel, H., and A. Morel, 1998: Light scattering and chlorophyll concentration in case 1 waters: A reexamination. Limnol. Oceanogr., 43, 847-858.

Lundgren, B., and N. K. Højerslev, 1971: Daylight measurements in the Sargasso Sea: Results from the "Dana" expedition January-April 1966. Institute of Physical Oceanography Rep. 14, University of Copenhagen, $44 \mathrm{pp}$.

Maffione, R. A., 2001: Evolution and revolution in measuring ocean optical properties. Oceanography, 14, 9-14.

Meyzonnette, J.-L., and T. Lépine, 2003: Bases de Radiometrie Optique. 2nd ed. Cépaduès-Editions, $252 \mathrm{pp}$.

Miller, R. L., M. Belz, C. Del Castillo, and R. Trzaska, 2002: Determining CDOM absorption spectra in diverse coastal environments using a multiple pathlength, liquid core waveguide system. Cont. Shelf Res., 22, 1301-1310, doi:10.1016/S02784343(02)00009-2.

Mobley, C. D., 1994: Light and Water: Radiative Transfer in Natural Waters. Elsevier, 592 pp.

—_, and Coauthors, 1993: Comparison of numerical models for computing underwater light fields. Appl. Opt., 32, 7484 7504.

Morel, A., 1974: Optical properties of pure water and pure seawater. Optical Aspects of Oceanography, N. G. Jerlov and E. Steeman Nielsen, Eds., Academic Press, 1-24.

— Limnol. Oceanogr., 22, 709-722.

_ , and B. Gentili, 1991: Diffuse reflectance of oceanic waters: Its dependence on sun angle as influenced by molecular scattering contribution. Appl. Opt., 30, 4427-4438.

$\longrightarrow$, and 1993: Diffuse reflectance of oceanic waters. 2. Bidirectional aspects. Appl. Opt., 32, 6864-6872.

— Implication of bidirectionality for the remote-sensing problem. Appl. Opt., 35, 4850-4862.

$\longrightarrow$, and — 2004: Radiation transport within oceanic (case 1) waters. J. Geophys. Res., 109, C06008, doi:10.1029/ 2003JC002259.

— , and - 2009: A simple band ratio technique to quantify the colored dissolved and detrital organic material from ocean color remotely sensed data. Remote Sens. Environ., 113, 998 1011, doi:10.1016/j.rse.2009.01.008.

_ - K. J. Voss, and B. Gentili, 1995: Bidirectional reflectance of oceanic waters: A comparison of modeled and measured upward radiance fields. J. Geophys. Res., 100, 13 143-13 150.

_ B. Gentili, H. Claustre, M. Babin, A. Bricaud, J. Ras, and F. Tieche, 2007: Optical properties of the "clearest" natural waters. Limnol. Oceanogr., 52, 217-229.

Morrow, J. H., S. B. Hooker, C. R. Booth, G. Bernhard, R. N. Lind, and J. W. Brown, 2010: Advances in measuring the apparent optical properties (AOPs) of optically complex waters. NASA GSFC Tech. Memo. NASA/TM-2010-215856, 80 pp.

Mueller, J. L., and Coauthors, 2003: Ocean optics protocols for satellite ocean color sensor validation, revision 4. Vol. 3, Radiometric Measurements and Data Analysis Protocols, NASA/TM-2003-21621/Rev, NASA Goddard Space Flight Center, 78 pp.
Pettersson, H., 1938: Measurements of the angular distribution of submarine light. Rapp. P.-V. Reun. - Cons. Int. Explor. Mer, 108, 7-12.

Plass, G. N., and G. W. Kattawar, 1969: Radiative transfer in an ocean-atmosphere system. Appl. Opt., 8, 455-466.

Pope, R. M., and E. S. Fry, 1997: Absorption spectrum (380$700 \mathrm{~nm}$ ) of pure water, II, integrating cavity measurements. Appl. Opt., 36, 8710-8723.

Preisendorfer, R. W., 1961: Application of radiative transfer theory to light measurements in the sea. Symposium on Radiant Energy in the Sea, IUGG Monogr., No. 10, L'Institut Géographique National, 11-30.

Prieur, L., and A. Morel, 1971: Etude theoretique du regime asymptotique: Relations entre caracteristiques optiques et coefficient $\&$ extinction relative a la penetration de la lumiere du jour. Cah. Oceanogr., 23, 35-48.

Sasaki, T., S. Watanabe, G. Oshiba, and N. Okami, 1958: Measurements of angular distribution of submarine daylight by means of a new instrument. J. Oceanogr. Soc. Japan, 14, 47-52.

,,,$----\ldots$, and M. Kajihara, 1962: On the instrument for measuring angular distribution of underwater radiance. Bull. Japan. Soc. Sci. Fish, 28, 489-496.

Smith, R. C., 1974: Structure of solar radiation in the upper layers of the sea. Optical Aspects of Oceanography, N. G. Jerlov and E. Steemann Nielsen, Eds., Academic Press, 95-119.

_ diance distribution camera system. Appl. Opt., 9, 2015-2022, doi:10.1364/AO.9.002015.

Thuillier, G., M. Hersé, P. C. Simon, D. Labs, H. Mandel, D. Gillotay, and T. Foujols, 1998: Observation of the visible solar spectral irradiance between 350 and $850 \mathrm{~nm}$ during the ATLAS I mission by the SOLSPEC spectrometer. Sol. Phys., 177, 41-61.

Tyler, J. E., 1960: Radiance distribution as a function of depth in an underwater environment. Bull. Scripps Inst. Oceanogr., 7, 363-411.

Voss, K. J., 1989a: Electro-optic camera system for measurement of the underwater radiance distribution. Opt. Eng., 28, 384-387.

_ 1989b: Use of the radiance distribution to measure the optical absorption coefficient in the ocean. Limnol. Oceanogr., 34, 1614-1622, doi:10.4319/lo.1989.34.8.1614.

_ , and G. Zibordi, 1989: Radiometric and geometric calibration of a visible spectral electro-optic fisheye camera radiance distribution system. J. Atmos. Oceanic Technol., 6, 652-662.

, and A. L. Chapin, 1992: Next-generation in-water radiance distribution camera system. Ocean Optics XI, G. D. Gilbert, Ed., International Society for Optical Engineering (SPIE Proceedings, Vol. 1750), 384-387.

— , and —_, 2005: Upwelling radiance distribution camera system, NURADS. Opt. Express, 13, 4250-4262, doi:10.1364/ OPEX.13.004250.

_ , and A. Morel, 2005: Bidirectional reflectance function for oceanic waters with varying chlorophyll concentration measurements versus predictions. Limnol. Oceanogr., 50, 698-705. , and N. Souaidia, 2010: POLRADS: Polarization radiance distribution measurement system. Opt. Express, 18, 19672 19680.

— A. Morel, and D. Antoine, 2007: Detailed validation of the bidirectional effect in various case 1 waters for application to ocean color imagery. Biogeosciences, 4, 781-789.

You, Y., G. W. Kattawar, K. J. Voss, P. Bhandari, J. Wei, M. R. Lewis, C. J. Zappa, and H. Schultz, 2011: Polarized light field under dynamic ocean surfaces: Numerical modeling compared 
with measurements. J. Geophys. Res., 116, C00H05, doi:10.1029/2011JC007278.

Zaneveld, R. J. V., 1974: New developments of the theory of radiative transfer in the oceans. Optical Aspects of Oceanography, N. G. Jerlov and E. Steemann Nielsen, Eds., Academic Press, 121-133.

, 1989: An asymptotic closure theory for irradiance in the sea and its inversion to obtain the inherent optical properties. Limnol. Oceanogr., 34, 1442-1452.

- and H. Pak, 1972: Some aspects of the axially symmetric submarine light field. J. Geophys. Res., 77, 2677-2680.
Zibordi, G., and K. J. Voss, 1989: Geometrical and spectral distribution of sky radiance: Comparison between simulations and field measurements. Remote Sens. Environ., 27, 343-358.

, D. D'Alimonte, and J.-F. Berthon, 2004: An evaluation of depth resolution requirements for optical profiling in coastal waters. J. Atmos. Oceanic Technol., 21, 1059-1073.

- J.-F. Berthon, F. Mélin, and D. D'Alimonte, 2011: Cross-site consistent in situ measurements for satellite ocean color applications: The BiOMaP radiometric dataset. Remote Sens. Environ., 115, 2104-2115. 